\title{
CLIMATIC DATA FOR WILLIAMS LAKE, HUBBARD COUNTY, MINNESOTA, 1983
}

By

A.M. Sturrock, D.O. Rosenberry, L.G. Engelbrecht, W.A. Gothard, and T.C. Winter

DEPARTMENT OF THE INTERIOR U.S. GEOLOGICAL SURVEY

Open-File Report 84247 


\section{UNITED STATES DEPARTMENT OF THE INTERIOR}

WILLIAM P. CLARK, Secretary

GEOLOGICAL SURVEY

Dallas L. Peck, Director

For additional information write to:

Thomas C. Winter

U.S. Geological Survey, MS 413

Box 25046, Denver Federal Center

Denver, CO 80225
For sale by:

Open-File Services Section Western Distribution Branch U.S. Geological Survey, MS 306 Box 25425, Federal Center Denver, CO 80225

Telephone: (303) 234-5888 
Page

Abstract

Introduction

7

Data collection and presentation 8

Acknowledgements

Reference

10

Tables

11 


\section{TABLES}

Table 1. Raft station--daily averages: (1) Lake-surface water

temperature; (2) dry- and wet-bulb air

temperatures; (3) vapor pressures; (4) Bowen

ratio; and (5) wind speed at 1,2 , and 4 meters.

Land station: (1) Daily average dry- and wet-bulb

air temperatures; (2) daily average vapor

pressure; (3) daily average wind speed at 2

meters; (4) daily total precipitation; and (5)

daily total short- and long-wave radiation. . . . . . . 11

2. Raft station: (1) Daily average lake-surface water

temperature; (2) daily average dry- and wet-bulb

air temperatures; and (3) daily maximum and

minimum dry-bulb air temperatures and the time

they occurred . . . . . . . . . . . . . . 21

3. Raft station: (1) Daily average wind speed at three

levels above the lake surface; and (2) daily

maximum and minimum wind speed at each level and

the time they occurred. . . . . . . . . . . . . 24

4. Land station: (1) Daily average dry- and wet-bulb air

temperatures; and (2) daily maximum and minimum

dry-bulb air temperatures and the time they

occurred. . . . . . . . . . . . . . . . . . . 33

5. Land station: (1) Daily average wind speed; (2) daily

maximum and minimum wind speed and the time they

occurred; and (3) daily total precipitation... . . . 36 
6. Land station: (1) Daily total short- and long-wave

radiation; (2) daily maximum short-wave radiation

and the time it occurred; and (3) daily maximum and

minimum long-wave radiation and the time they

occurred. . . . . . . . . . . . . . . . . . . 


\section{MULTIPLY}

centimeter

millibar

millibar

mile per hour

calories per square

centimeter per day

calories per square

centimeter per day
$\underline{B Y}$

.394

.0145

1.0197

1.609

278.96

25.913

${ }^{\circ} \mathrm{C}$

C CONVERSION EQUATION FOR DEGREES FAHRENHEIT TO CENTIGRADE:

${ }^{\circ} \mathrm{C}=5 / 9\left({ }^{\circ} \mathrm{F}-32\right)$ or ${ }^{\circ} \mathrm{F}=9 / 5\left({ }^{\circ} \mathrm{C}\right)+32$

TO OBTAIN

inch

pounds per square inch

grams per square centimeter

kilometer per hour

watts per square meter

watts per square foot 


\section{Climatic data for Williams Lake, Hubbard County, Minnesota}

1983

by

A. M. Sturrock, D. O. Rosenberry, L. G. Engelbrecht,

W. A. Gothard, and T. C. Winter

\section{ABSTRACT}

Research on the hydrology of Williams Lake, north-central Minnesota includes study of evaporation. Presented here are those climatic data needed for energy-budget and mass-transfer studies, including: water-surface temperature, dry-bulb and wet-bulb air temperatures, wind speed, precipitation, and solar radiation. Data are collected at raft and land stations.

\section{INTRODUCTION}

Climatic data are being collected at Williams Lake, Hubbard County, Minnesota, as part of a continuing study by the U.S. Geological Survey of the hydrology of the lake. Williams lake is one of several lakes in different parts of the United States that have been selected for intensive study of hydrological and related chemical and biological processes. The rationale for selection of Williams Lake is given by Siegel and Winter (1980). 
Data presented here are being collected principally for studies of evaporation. Therefore, the period of record includes only the time when the lake is ice-free. The period of record for 1983 is from May 30 (Julian day 150) through October 26 (Julian day 299), 1983. Although data were collected before and after these dates (the raft was placed on the lake May 5), the interval from day 150 to 299 is the part of the year for which evaporation will be determined by the energy-budget method. Within each table, the data are grouped into energy-budget periods; the periods are defined by the dates thermal surveys were made in the lake. For example; the first energy-budget period is day 150 through day 167.

Climatic instruments are located on a raft in the middle of the lake and at a land station. Instruments on the raft include anemometers at 1 ; 2 , and 4 meters above the water surface, a thermistor psychrometer with wet and dry-bulb temperature sensors fixed at 2 meters above the water surface, and a water-temperature sensor located beneath the raft at a water depth of about 1 centimeter. Data from the above sensors are recorded by a Campbell CR-21 $1 /$ digital data logger located on the raft. The data logger scans the sensors every minute and calculates an hourly average. In addition; for selected sensors, maximum and minimum values and the time they occur are saved and recorded at midnight of each day. Additional analog instruments for measuring water-surface temperature and wind speed also are located on the raft. These are used to backup the primary instruments for quality control and for filling in missing data.

1/ The use of brand names in this report is for identification only, and does not constitute endorsement by the U.S. Geological Survey. 
The land station consists of long- and short-wave radiometers, an anemometer at 2 meters above the land surface, a thermistor psychrometer also at 2 meters above land surface, and a tipping-bucket rain gage. These data also are recorded by a digital data logger that operates similarly to that on the raft. Backup instruments include an analog hygrothermograph, a digital hygrothermograph, and a manually-read rain gage.

Calibration checks with independent laboratory thermometers and motorized psychrometers are made every few days at both stations.

Data presented here are daily summaries. For periods that the primary instruments were not operating properly, daily values were obtained by regression using data from backup instruments, provided a good statistical relationship could be established. Data used to establish regressions were selected so they bracketed the period of missing or inadequate data. Only table 1 , which is considered to be the primary source of data for evaporation studies, includes values obtained by regression. These are noted by footnotes, and the regressions referred to are as follows:
REGRESSION 1
DATA POINTS
$\mathrm{R}^{2}$ VALUE
$Y=0.641+0.959 x$
0.989

REGRESSION 2
$Y=-1.020+1.354 x$
23
0.913

Vapor pressure of water $\left(e_{0}\right)$ is calculated using water-temperature data and assuming the air is completely saturated at the air-water interface. 
Although only daily values are reported here, hourly values also were recorded. Hourly values are voluminous and expensive to reproduce, but they are available for all or part of the period of record on request.

\section{ACKNOWLEDGEMENTS}

We are especially indebted to Ken Chase for allowing us to place climatic instruments on his property. The Minnesota Department of Natural Resources, Division of Waters, provided funding for part of the instrumentation at Williams Lake.

\section{REFERENCE}

Slegel, D. I. and Winter, T. C., 1980, Hydrologic Setting of Williams Lake, Hubbard County, Minnesota: U.S. Geological Survey Open-File Report $80-403,56$ p. 

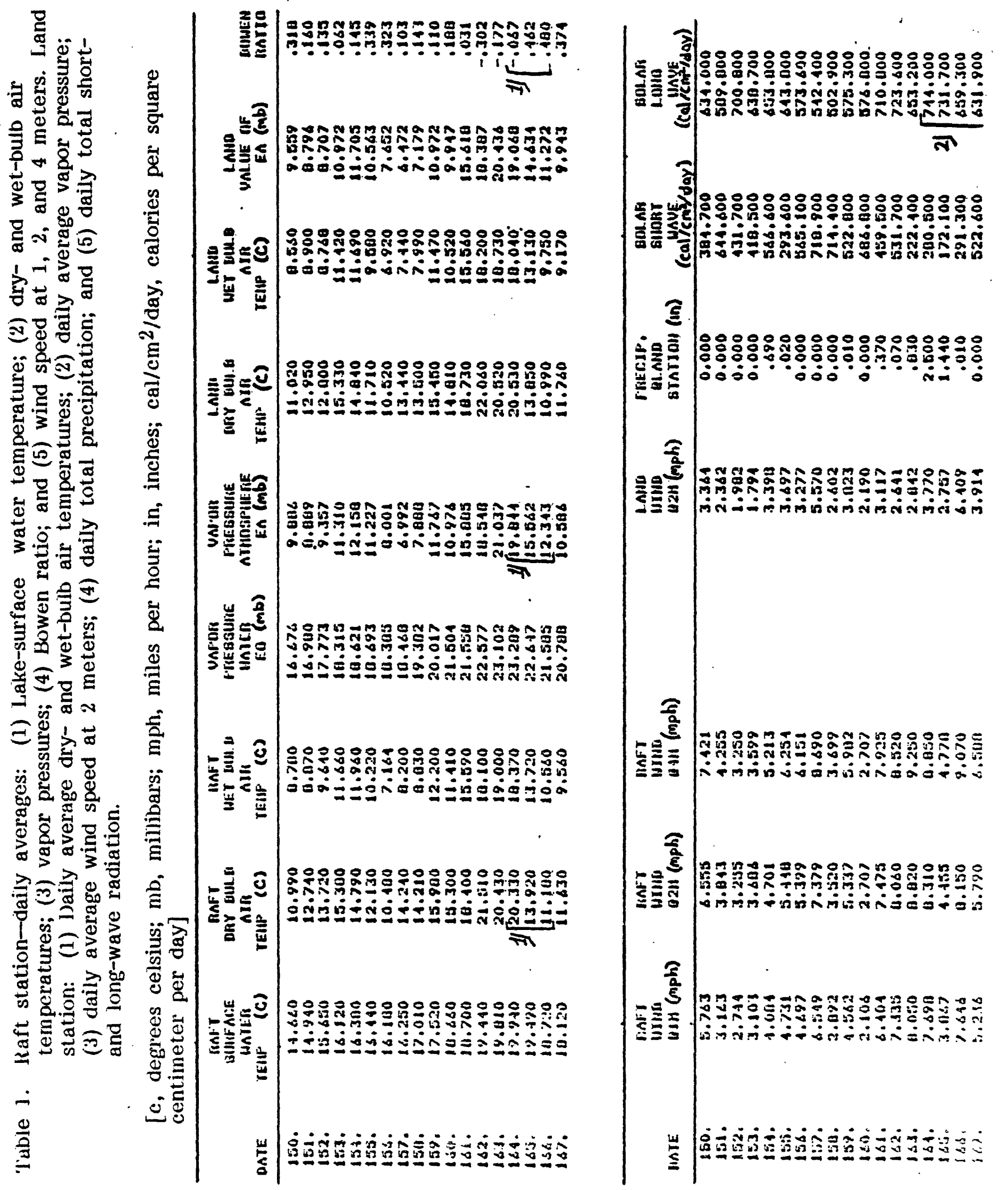


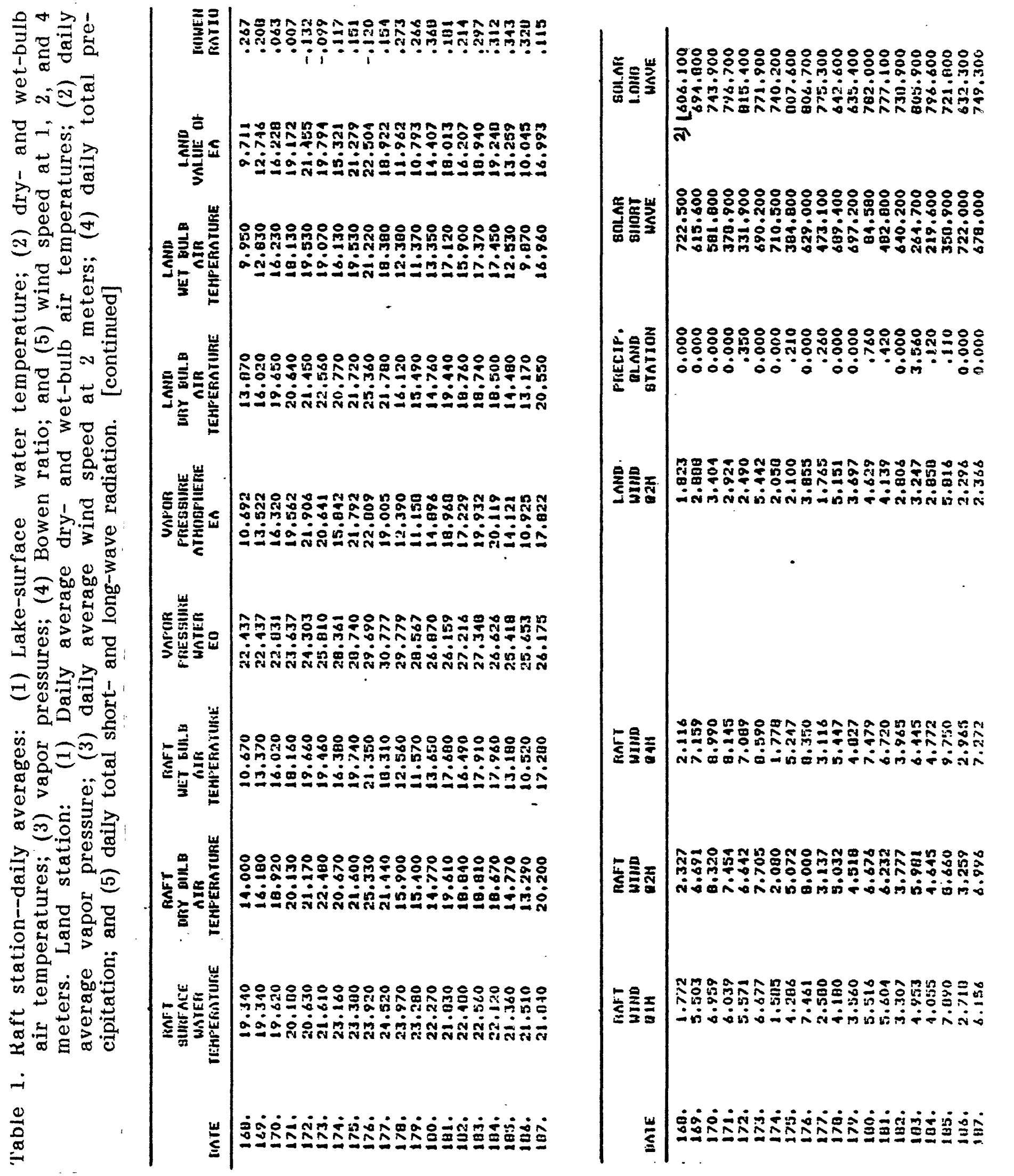




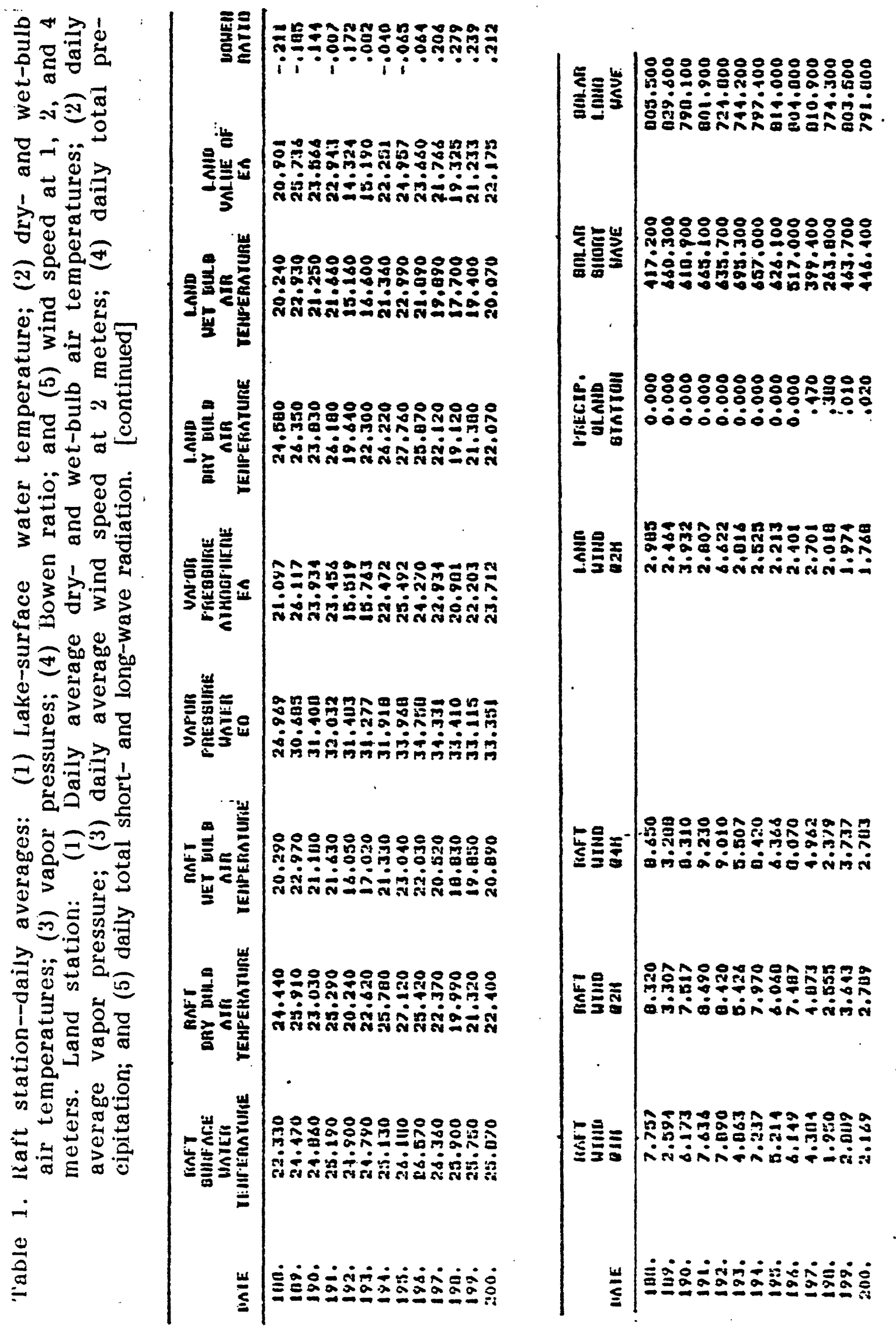




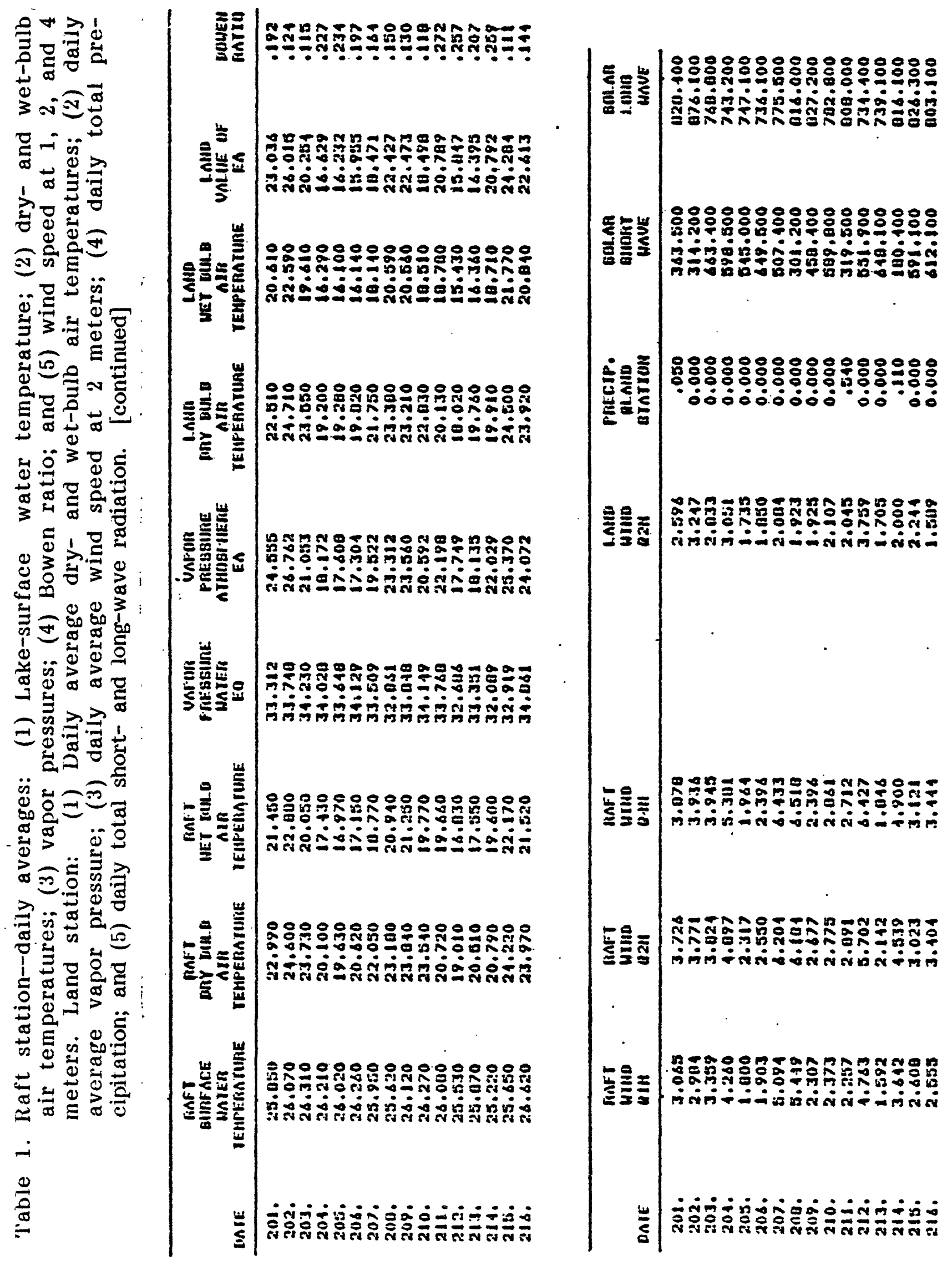




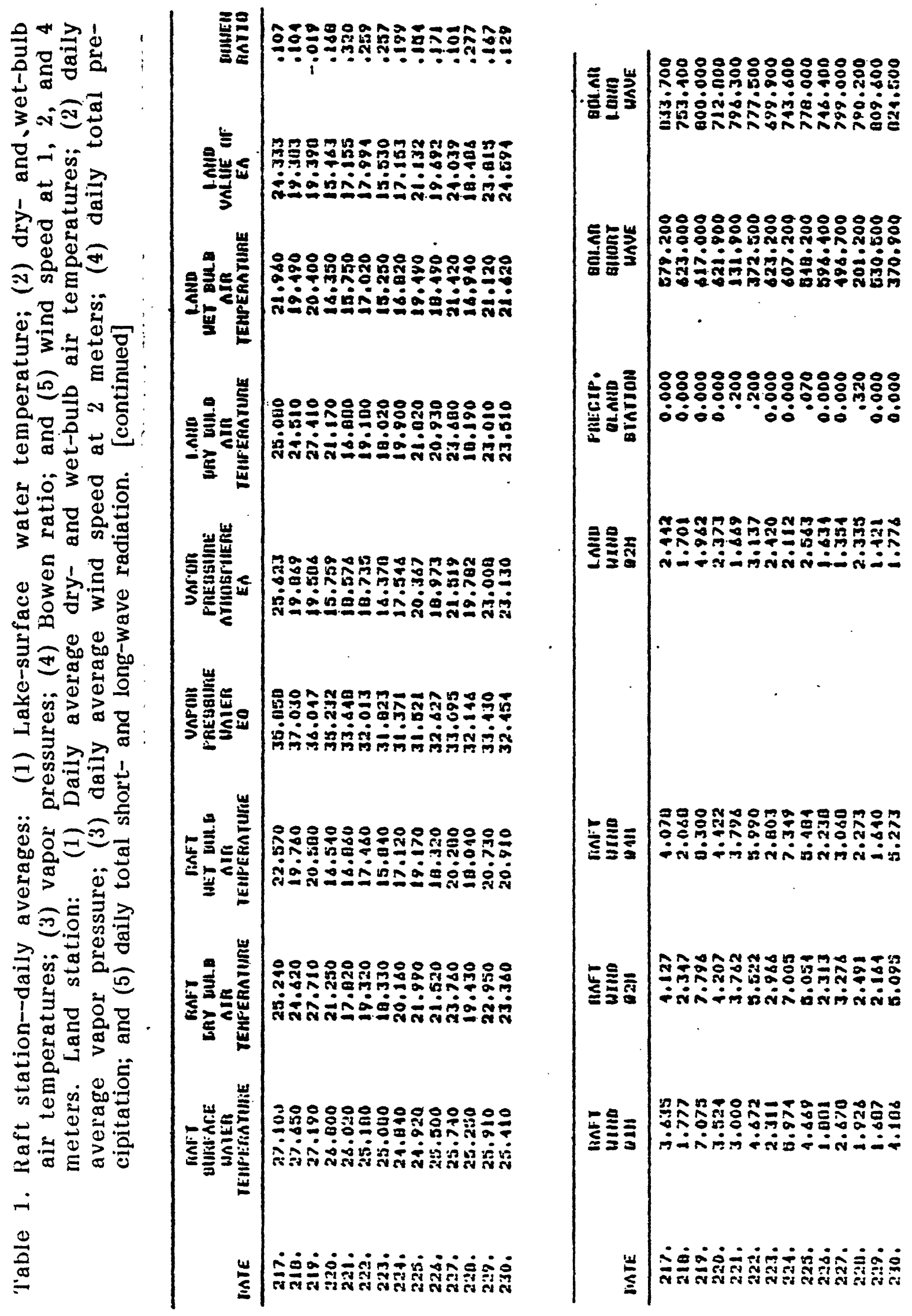



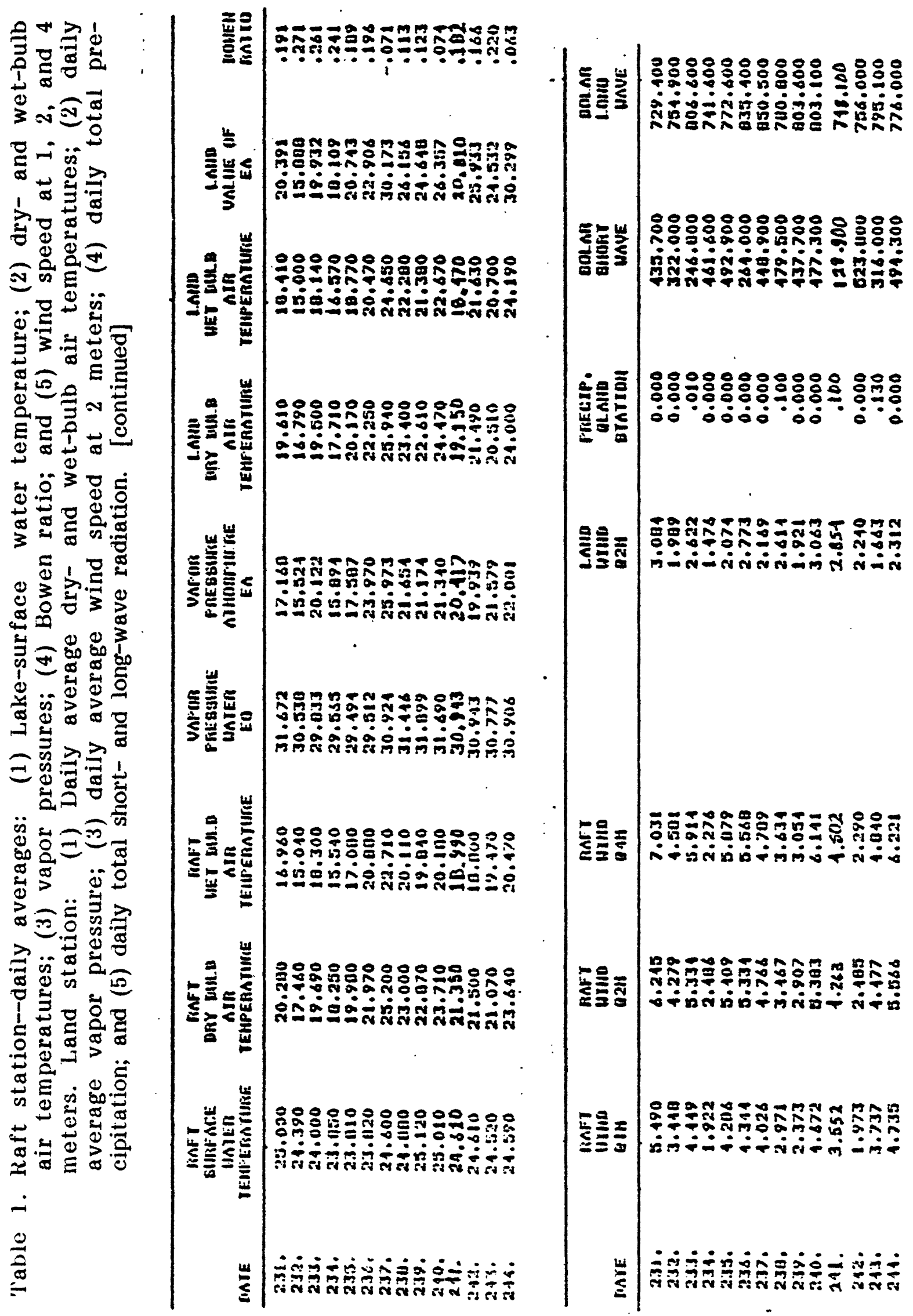


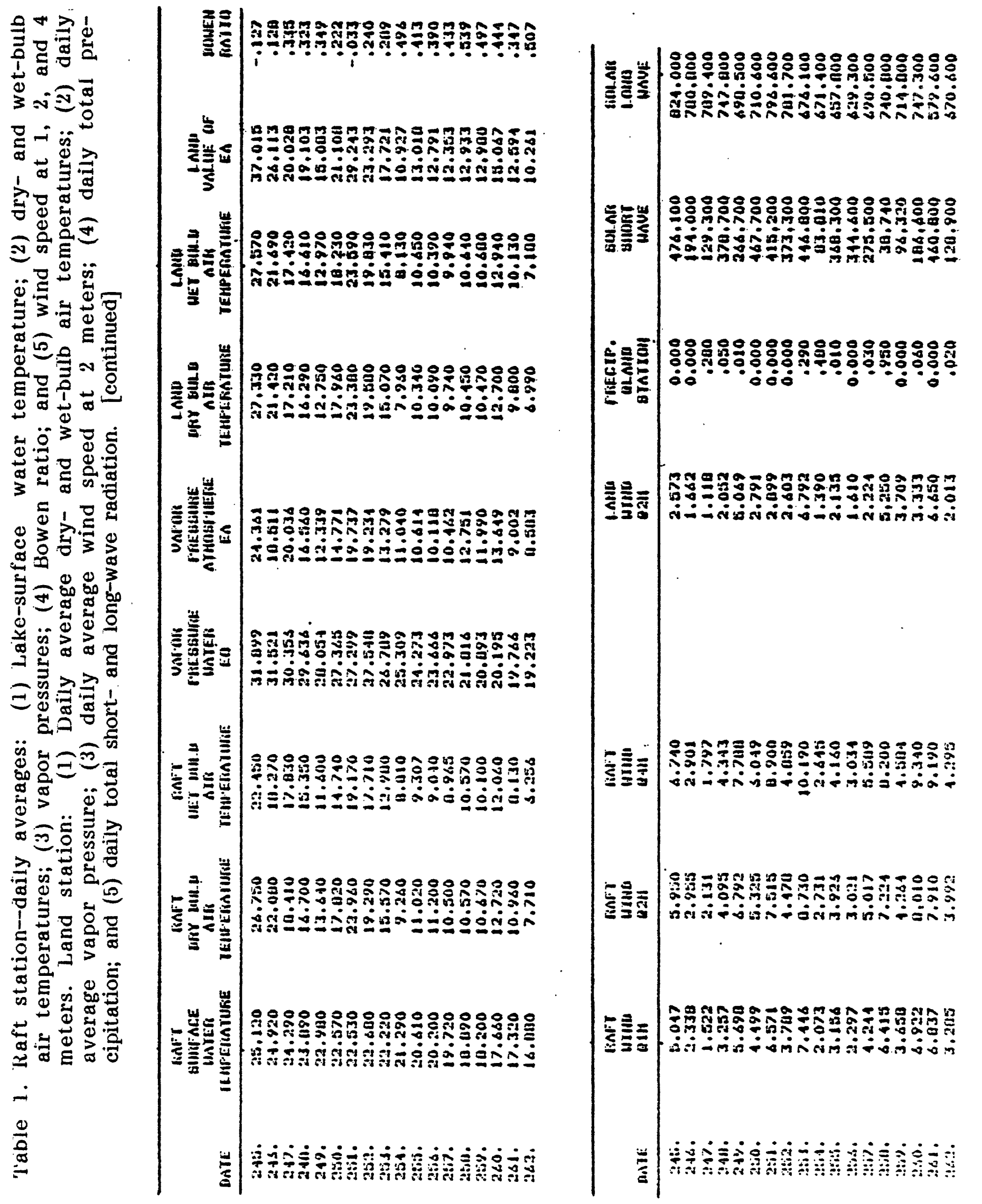




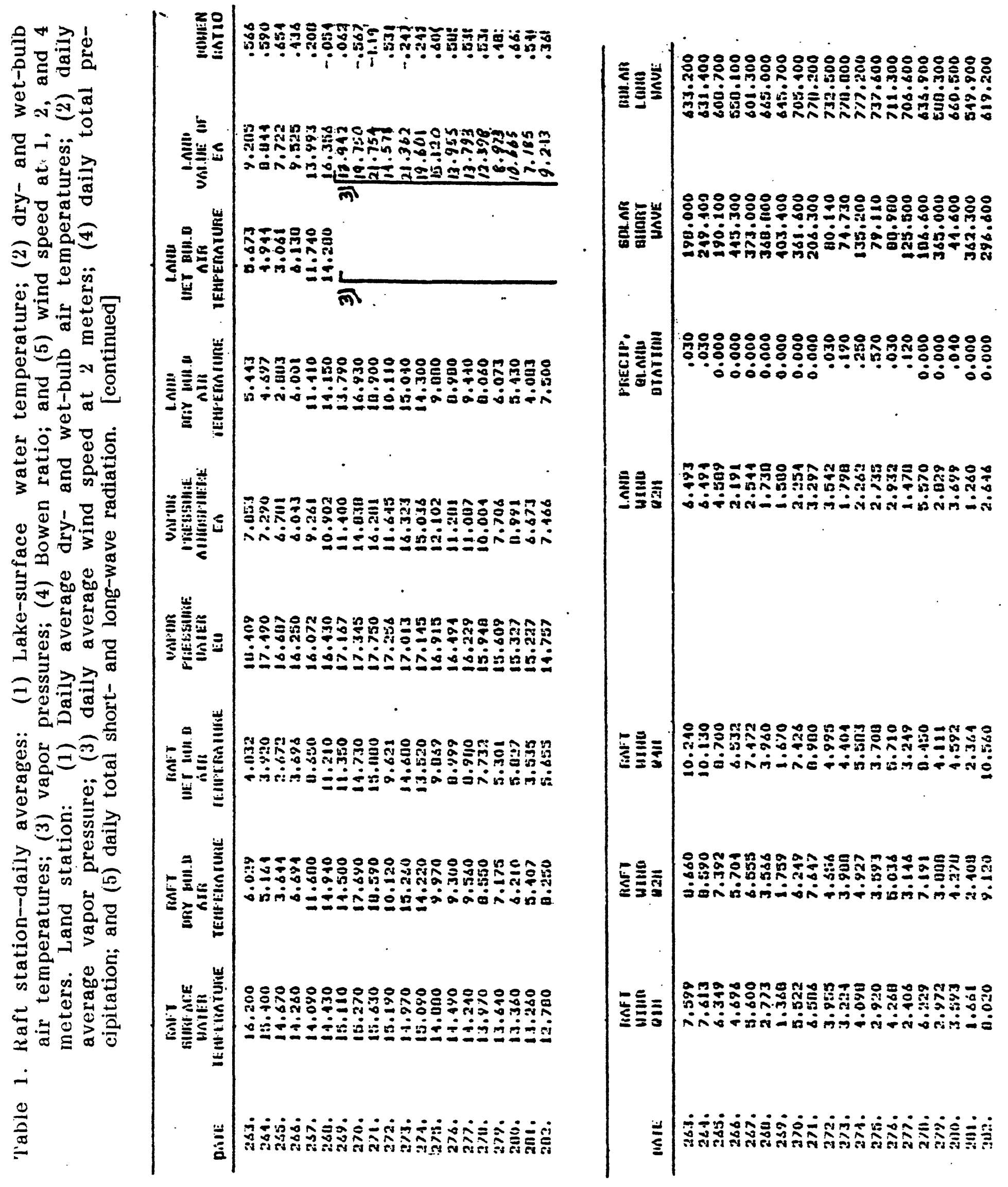




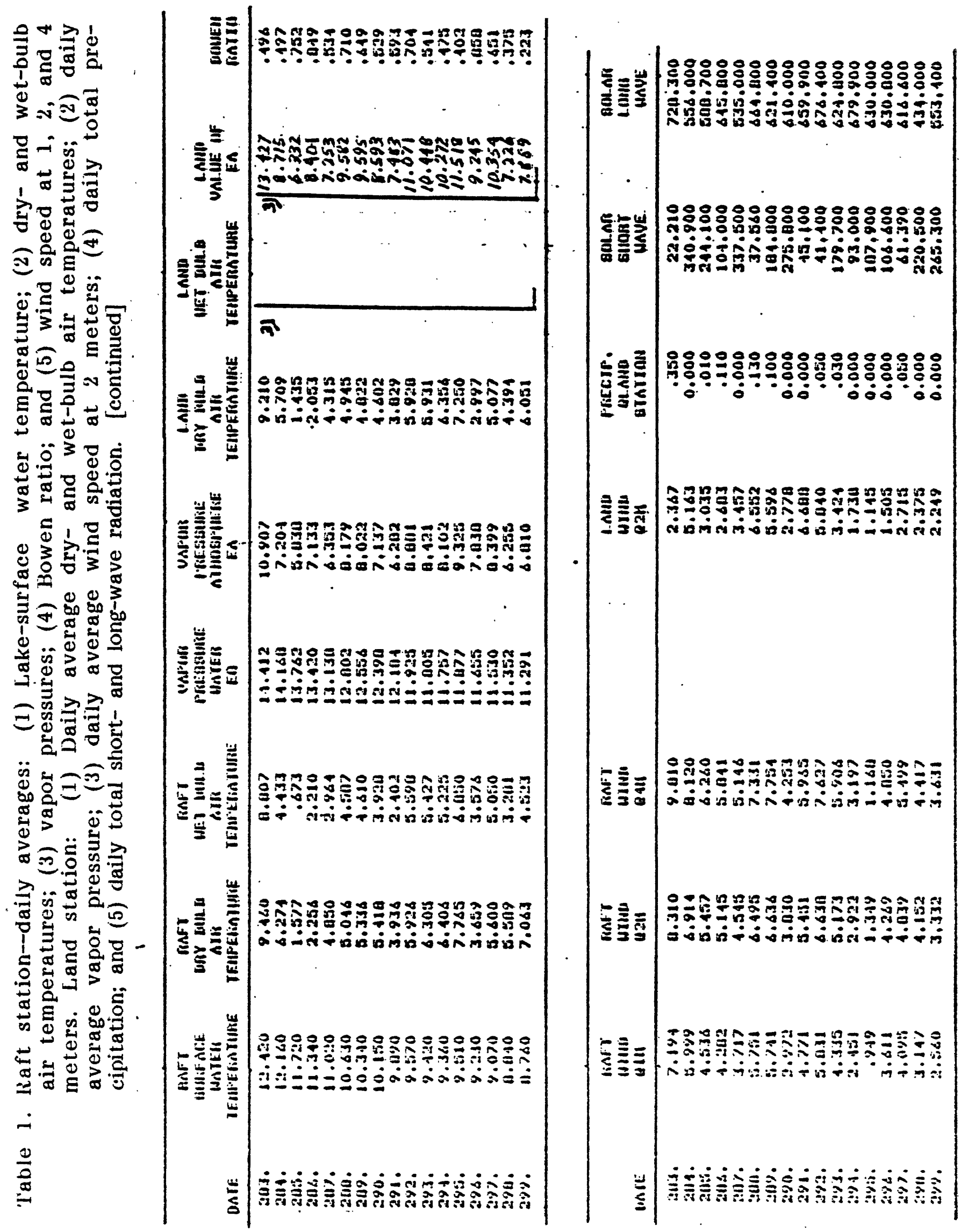


1/ Values of raft dry-bulb for days 164 through 166 were found by linear regression with back-up instruments (regression 1). Values of ea raft, and the Bowen ratio for corresponding days had to be recalculated due to their dependence on the raft dry-bulb values.

2/ Solar long-wave radiation for days 164 through 168 was obtained using Brunt's equation.

3/ Values of land ea for days 269 through 299 were found by linear regression with back-up instruments (regression 2). Land wet-bulb values were lost due to a dry instrument reservoir. 
T'able 2. Raft station: (1) Daily average lake-surface water temperature; (2) daily average dry- and wet-bulb air temperatures; and (3) daily maximum and minimum dry-bulb air temperatures and the time they occurred.

[c, degrees celsius]

\begin{tabular}{|c|c|c|c|c|c|c|c|}
\hline Inte & $\begin{array}{l}\text { SURFHCE } \\
\text { WATER (C) }\end{array}$ & $\begin{array}{c}\text { DRY BULES } \\
\text { AIR (E) }\end{array}$ & $\begin{array}{l}\text { MaXIYYUY } \\
\text { hIF: (e) }\end{array}$ & $\begin{array}{l}\text { TIME OF } \\
\text { MAXIMUM }\end{array}$ & $\begin{array}{l}\text { MENIMUJM } \\
\text { AIR (C) }\end{array}$ & $\begin{array}{l}\text { TIME of } \\
\text { MINIMLHY }\end{array}$ & $\begin{array}{l}\text { WET BUR } \\
\text { AIR (C) }\end{array}$ \\
\hline 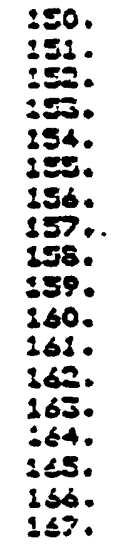 & $\begin{array}{l}14.66 \\
14.94 \\
15.65 \\
16.12 \\
16.38 \\
16.44 \\
16.18 \\
16.25 \\
17.01 \\
17.52 \\
18.66 \\
18.70 \\
19.44 \\
14.51 \\
19.94 \\
19.49 \\
18.72 \\
18.12\end{array}$ & $\begin{array}{l}10.99 \\
: 2.74 \\
: 3.72 \\
13.38 \\
14.79 \\
22.13 \\
10.48 \\
14.24 \\
14.21 \\
15.98 \\
15.30 \\
18.40 \\
21.31 \\
20.43\end{array}$ & $\begin{array}{l}14.19 \\
19.50 \\
18.85 \\
20.34 \\
19.64 \\
15.77 \\
14.62 \\
20.62 \\
22.57 \\
20.78 \\
29.98 \\
26.24 \\
25.44 \\
29.10\end{array}$ & $\begin{array}{l}1908 . \\
1806 . \\
1749 . \\
1738 . \\
1852 . \\
16160 \\
1740 . \\
1626 . \\
1834 . \\
1442 . \\
1523 . \\
1452 . \\
1620 . \\
2036 .\end{array}$ & $\begin{array}{r}7.86 \\
7.57 \\
7.42 \\
8.73 \\
16.75 \\
9.17 \\
5.92 \\
7.07 \\
3.99 \\
9.17 \\
6.54 \\
12.45 \\
17.00 \\
18.67\end{array}$ & $\begin{array}{r}429 . \\
429 . \\
509 . \\
419 . \\
2 ! 50 . \\
311 . \\
2305 . \\
180 \\
454 . \\
2530 \\
358 . \\
546 . \\
511 . \\
6 .\end{array}$ & $\begin{array}{r}5.73 \\
8.87 \\
9.64 \\
11.66 \\
11.98 \\
10.22 \\
7.16 \\
8.20 \\
8.83 \\
12.20 \\
11.41 \\
15.59 \\
18.10 \\
19.00\end{array}$ \\
\hline
\end{tabular}

\begin{tabular}{|c|c|c|c|c|c|c|c|}
\hline DnTE & $\begin{array}{c}\text { SURFACE } \\
\text { WATER }\end{array}$ & $\begin{array}{c}\text { ตRY gut } 3 \text { - } \\
\text { AIR }\end{array}$ & $\begin{array}{c}\text { MARIMUn } \\
\text { AIK }\end{array}$ & $\begin{array}{l}\text { TIME OF } \\
\text { MAXIMUH }\end{array}$ & $\begin{array}{c}\text { MINIMUY } \\
\text { AIR }\end{array}$ & $\begin{array}{l}\text { TIME OF } \\
\text { MINIMUH }\end{array}$ & $\begin{array}{c}\text { WET BULS } \\
\text { AIR }\end{array}$ \\
\hline $\begin{array}{l}368 . \\
269 . \\
170 . \\
171 . \\
172 . \\
175 . \\
174 . \\
175 . \\
176 . \\
177 . \\
178 . \\
: 79 . \\
180 . \\
181 . \\
185 . \\
183 . \\
184 . \\
295 . \\
165 . \\
187 .\end{array}$ & $\begin{array}{l}19.34 \\
19.34 \\
19.52 \\
20.18 \\
20.33 \\
29.61 \\
23.16 \\
23.38 \\
23.92 \\
24.52 \\
22.97 \\
23.39 \\
22.27 \\
21.83 \\
22.48 \\
22.36 \\
22.12 \\
21.36 \\
21.51 \\
21.84\end{array}$ & $\begin{array}{l}14.00 \\
16.18 \\
16.92 \\
20.13 \\
21.17 \\
22.48 \\
20.67 \\
21.60 \\
25.35 \\
21.44 \\
15.90 \\
15.40 \\
14.77 \\
19.61 \\
18.84 \\
13.81 \\
18.67 \\
14.77 \\
13.29 \\
20.20\end{array}$ & $\begin{array}{l}20.52 \\
20.79 \\
23.89 \\
22.81 \\
24.21 \\
23.62 \\
27.03 \\
27.29 \\
29.93 \\
25.09 \\
19.20 \\
22.10 \\
18.06 \\
23.16 \\
24.30 \\
24.39 \\
21.37 \\
29.11 \\
19.29 \\
26.76\end{array}$ & $\begin{array}{l}1=30 . \\
1220 . \\
1733 . \\
1334 . \\
1048 . \\
1031 . \\
1344 . \\
1510 . \\
1708 . \\
1405 . \\
1702 . \\
1545 . \\
2350 . \\
1003 . \\
1333 . \\
2520 \\
1136 . \\
1431 . \\
1313 . \\
2646 .\end{array}$ & $\begin{array}{r}5.92 \\
9.44 \\
12.95 \\
17.18 \\
17.79 \\
15.59 \\
13.57 \\
17.44 \\
20.17 \\
14.98 \\
11.63 \\
7.24 \\
13.22 \\
14.98 \\
14.10 \\
16.30 \\
15.35 \\
9.52 \\
6.54 \\
10.84\end{array}$ & $\begin{array}{r}406 . \\
244 . \\
443 . \\
229 . \\
913 . \\
2348 . \\
419 . \\
625 . \\
506 . \\
2329 . \\
2344 . \\
426 . \\
1124 . \\
2400 . \\
530 . \\
220 . \\
2400 . \\
2348 . \\
449 . \\
123 .\end{array}$ & $\begin{array}{l}10.67 \\
13.57 \\
16.02 \\
18.16 \\
19.66 \\
19.46 \\
16.35 \\
19.74 \\
21.35 \\
18.31 \\
19.56 \\
11.37 \\
13.05 \\
17.68 \\
13.49 \\
17.91 \\
17.96 \\
13.28 \\
10.52 \\
17.25\end{array}$ \\
\hline
\end{tabular}

\begin{tabular}{|c|c|c|c|c|c|c|c|}
\hline DATE & $\begin{array}{l}\text { SURFACE } \\
\text { WATER }\end{array}$ & $\begin{array}{c}\text { DRY BULB } \\
\text { AIR }\end{array}$ & $\begin{array}{c}\text { Maximum } \\
\text { AIก }\end{array}$ & $\begin{array}{l}\text { TIME OF } \\
\text { MAXIMUH }\end{array}$ & $\begin{array}{l}\text { AIHIMUM } \\
\text { AIR }\end{array}$ & $\begin{array}{l}\text { TIME OF } \\
\text { MINIMUK }\end{array}$ & WET BUL \\
\hline $\begin{array}{l}188 . \\
199 . \\
190 . \\
191 . \\
192 . \\
195 . \\
194 . \\
195 . \\
195 . \\
197 . \\
198 . \\
159 . \\
200 .\end{array}$ & $\begin{array}{l}22.53 \\
24.47 \\
24.86 \\
25.19 \\
24.90 \\
24.70 \\
25.10 \\
26.18 \\
20.57 \\
26.36 \\
20.90 \\
25.75 \\
25.37\end{array}$ & $\begin{array}{l}24.44 \\
25.91 \\
23.03 \\
2=.29 \\
20.24 \\
20.62 \\
25.79 \\
27.09 \\
25.49 \\
=1.37 \\
19.99 \\
21.02 \\
=0.40\end{array}$ & $\begin{array}{l}29.84 \\
31.07 \\
27.82 \\
30.55 \\
20.00 \\
25.79 \\
30.63 \\
32.05 \\
28.05 \\
26.00 \\
25.80 \\
20.05 \\
20.05\end{array}$ & 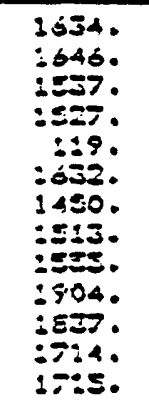 & $\begin{array}{l}20.25 \\
19.90 \\
19.20 \\
18.41 \\
: 5.50 \\
\vdots 3.25 \\
: 9.73 \\
20.28 \\
=2.54 \\
18.58 \\
16.21 \\
: 7.88 \\
17.55\end{array}$ & 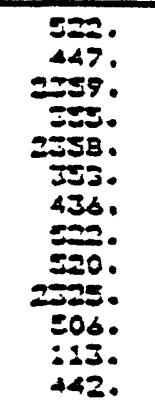 & $\begin{array}{l}20.29 \\
29.97 \\
21.18 \\
21.05 \\
20.05 \\
17.02 \\
31.35 \\
23.04 \\
21.03 \\
20.05 \\
18.83 \\
19.85 \\
20.89\end{array}$ \\
\hline
\end{tabular}


Table 2. Raft station: (1) Daily average lake-surface water temperature; (2) daily average dry- and wet-bulb air temperatures; and (3) daily maximum and minimum dry-bulb air temperatures and the time they occurred. [continued]

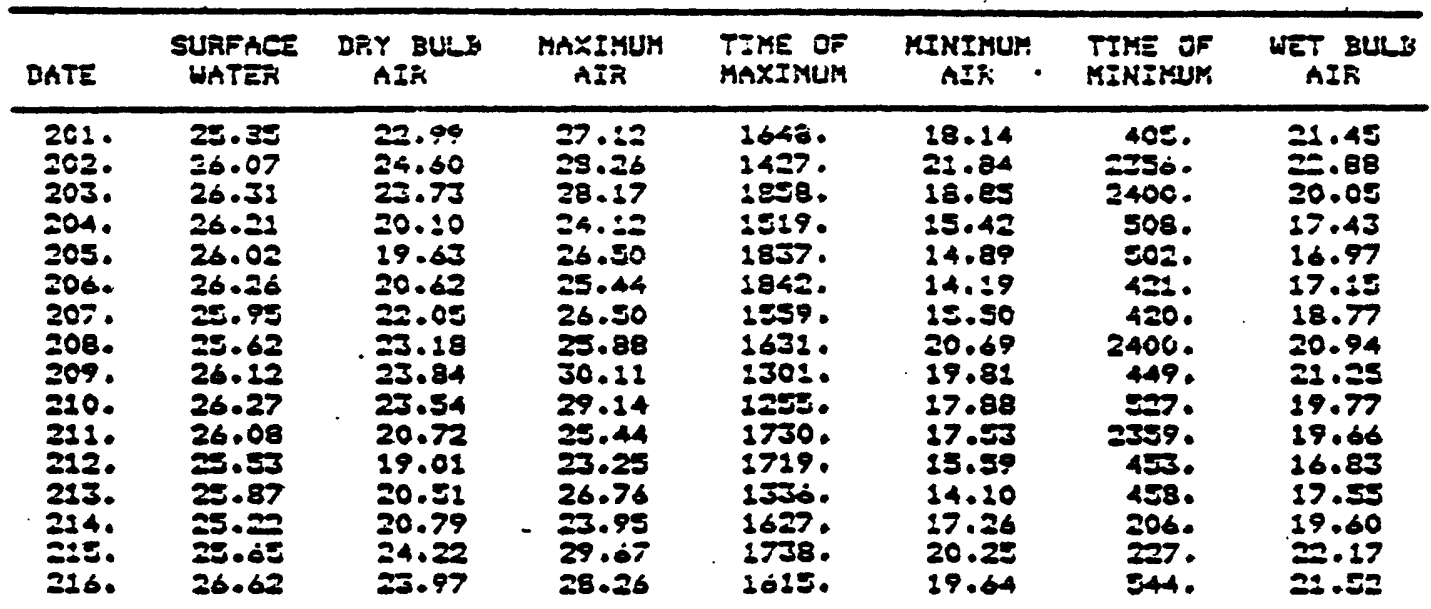

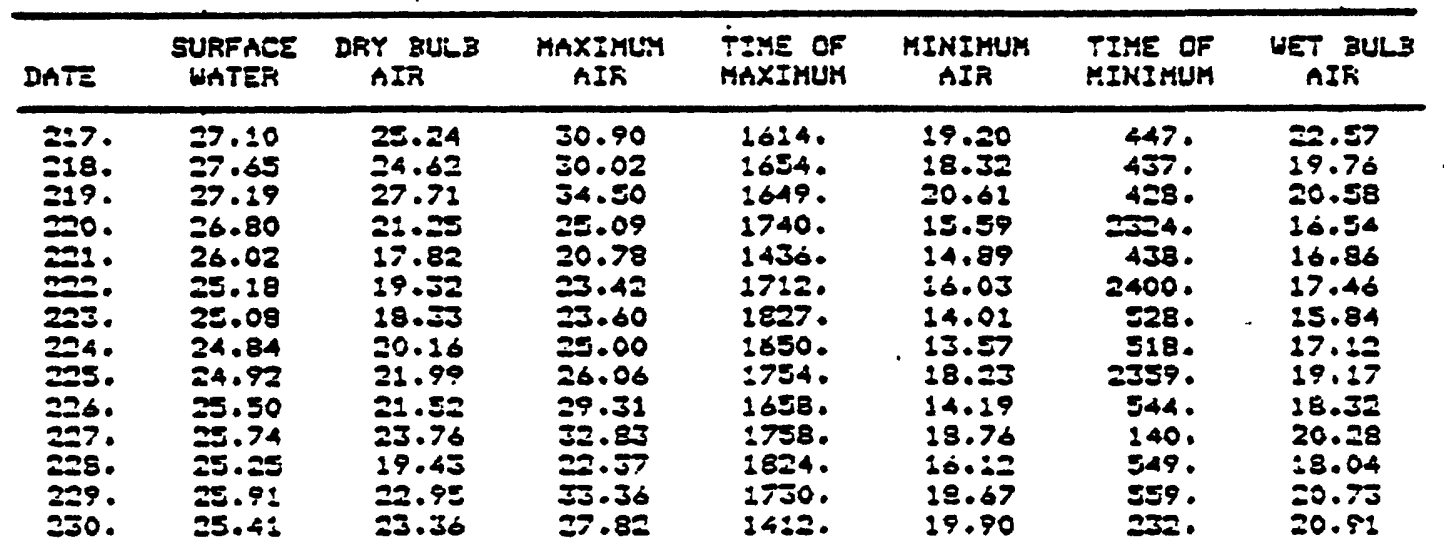

\begin{tabular}{|c|c|c|c|c|c|c|c|}
\hline DATE & $\begin{array}{c}\text { SURFACE } \\
\text { WATER }\end{array}$ & $\begin{array}{c}\text { DRY BULB } \\
\text { AIR }\end{array}$ & $\begin{array}{c}\text { MXIMUH } \\
\text { AIR }\end{array}$ & $\begin{array}{l}\text { PIHE OF } \\
\text { MAXIMUH }\end{array}$ & $\begin{array}{c}\text { MINIKUM } \\
\text { AIR }\end{array}$ & $\begin{array}{l}\text { TIHE OF } \\
\text { HINIMUH }\end{array}$ & WET EUL? \\
\hline $\begin{array}{l}232 . \\
232 . \\
233 . \\
234 . \\
235 . \\
236 . \\
237 . \\
238 . \\
258 . \\
240 . \\
241 . \\
242 . \\
245 . \\
244 .\end{array}$ & $\begin{array}{l}25.00 \\
24.39 \\
24.00 \\
23.85 \\
23.81 \\
23.82 \\
24.60 \\
24.88 \\
25.22 \\
25.01 \\
24.61 \\
24.61 \\
24.52 \\
24.59\end{array}$ & $\begin{array}{l}20.93 \\
17.46 \\
19.69 \\
18.25 \\
19.98 \\
21.97 \\
25.20 \\
23.00 \\
23.27 \\
=3.71 \\
21.35 \\
2: .50 \\
=2.07 \\
=3.04\end{array}$ & $\begin{array}{l}24.04 \\
22.63 \\
25.18 \\
23.33 \\
23.95 \\
26.06 \\
31.16 \\
25.43 \\
29.03 \\
30.19 \\
25.36 \\
23.00 \\
25.97 \\
20.19\end{array}$ & $\begin{array}{l}1409 . \\
1608 . \\
1224 . \\
1516 . \\
1640 . \\
1641 . \\
1622 . \\
1526 . \\
1355 . \\
1645 . \\
1452 . \\
1725 . \\
1258 . \\
1515 .\end{array}$ & $\begin{array}{l}12.95 \\
12.55 \\
15.77 \\
13.13 \\
14.80 \\
19.02 \\
19.29 \\
17.79 \\
16.91 \\
17.79 \\
18.24 \\
17.09 \\
17.44 \\
17.00\end{array}$ & $\begin{array}{r}2354 . \\
=38 . \\
2355 . \\
541 . \\
201 . \\
529 . \\
427 . \\
547 . \\
510 . \\
530 . \\
9: 7 . \\
5=8 . \\
556 . \\
50 .\end{array}$ & $\begin{array}{l}16.90 \\
15.04 \\
18.30 \\
15.54 \\
17.08 \\
20.83 \\
25.71 \\
20.21 \\
29.84 \\
25.18 \\
18.99 \\
: 8.80 \\
19.47 \\
20.47\end{array}$ \\
\hline
\end{tabular}


Table 2. Raft station: (1) Daily average lake-surface water temperature; (2) daily average dry- and wet-bulb air temperatures; and (3) daily maximum and minimum dry-bulb air temperatures and the time they occurred. [continued]

\begin{tabular}{|c|c|c|c|c|c|c|c|}
\hline DATE & $\begin{array}{l}\text { SURFACE } \\
\text { WATER }\end{array}$ & อกY & $\begin{array}{c}\text { MAXIMU: } \\
\text { AIก }\end{array}$ & $\begin{array}{l}\text { IIME of } \\
\text { HAXIHUH }\end{array}$ & $\begin{array}{c}\text { MINIMUA } \\
\text { AIR }\end{array}$ & $\begin{array}{l}\text { TIME OF } \\
\text { MINEMUM }\end{array}$ & $\begin{array}{l}\text { WET EULB } \\
\text { AIR }\end{array}$ \\
\hline $\begin{array}{l}245 . \\
246 . \\
247 . \\
242 . \\
249 . \\
250 . \\
251 . \\
252 . \\
253 . \\
254 . \\
255 . \\
256 . \\
257 . \\
258 . \\
259 . \\
260 . \\
261 . \\
262 .\end{array}$ & $\begin{array}{l}25.12 \\
24.92 \\
24.29 \\
23.89 \\
22.99 \\
22.57 \\
22.53 \\
22.08 \\
22.02 \\
21.29 \\
20.61 \\
20.20 \\
19.72 \\
18.89 \\
18.20 \\
17.60 \\
17.38 \\
16.88\end{array}$ & $\begin{array}{l}26.75 \\
29.08 \\
25.41 \\
10.70 \\
13.64 \\
17.52 \\
20.96 \\
19.29 \\
15.57 \\
9.26 \\
11.02 \\
11.20 \\
10.50 \\
10.57 \\
10.07 \\
12.72 \\
10.96 \\
7.71\end{array}$ & $\begin{array}{l}35.71 \\
25.80 \\
33.42 \\
22.63 \\
26.12 \\
22.82 \\
28.96 \\
23.51 \\
19.64 \\
12.16 \\
14.99 \\
35.68 \\
25.15 \\
11.55 \\
12.87 \\
15.33 \\
14.62 \\
9.61\end{array}$ & $\begin{array}{r}1443 . \\
8 . \\
1154 . \\
1349 . \\
1054 . \\
16 \vdots 3 . \\
1934 . \\
1506 . \\
341 . \\
1129 . \\
1540 . \\
1338 . \\
1310 . \\
3530 \\
1356 . \\
1434 . \\
1634 . \\
1309 .\end{array}$ & $\begin{array}{r}20.25 \\
17.79 \\
15.06 \\
12.16 \\
10.23 \\
13.75 \\
16.82 \\
16.82 \\
10.84 \\
6.45 \\
6.54 \\
6.71 \\
4.43 \\
9.52 \\
9.08 \\
10.23 \\
6.36 \\
5.57\end{array}$ & $\begin{array}{r}430 . \\
2358 . \\
2353 . \\
609 . \\
63: . \\
509 . \\
618 . \\
921 . \\
2359 . \\
527 . \\
542 . \\
2333 . \\
630 . \\
2359 . \\
431 . \\
333 . \\
2328 . \\
2322 .\end{array}$ & $\begin{array}{r}72.45 \\
13.27 \\
17.83 \\
13.95 \\
11.60 \\
14.74 \\
19.17 \\
17.71 \\
12.98 \\
8.81 \\
9.31 \\
9.04 \\
8.97 \\
10.57 \\
10.10 \\
12.06 \\
8.13 \\
8.26\end{array}$ \\
\hline
\end{tabular}

\begin{tabular}{|c|c|c|c|c|c|c|c|}
\hline DATE & $\begin{array}{c}\text { SURFACE } \\
\text { WATER }\end{array}$ & $\begin{array}{c}\text { DRY BULA } \\
\text { AIR }\end{array}$ & $\begin{array}{l}\text { MAXIMUM } \\
\text { AIR }\end{array}$ & $\begin{array}{l}\text { TIME of } \\
\text { MAXIMUH }\end{array}$ & $\begin{array}{c}\text { MINIMUH } \\
\text { AIR }\end{array}$ & $\begin{array}{l}\text { IIME OF } \\
\text { HINIMUY }\end{array}$ & $\begin{array}{c}\text { WET BULS } \\
\text { AIK }\end{array}$ \\
\hline $\begin{array}{l}263 . \\
264 . \\
265 . \\
260 . \\
267 . \\
268 . \\
265 . \\
270 . \\
271 . \\
272 . \\
273 . \\
274 . \\
275 . \\
276 . \\
277 . \\
278 . \\
279 . \\
290 . \\
281 . \\
222 .\end{array}$ & $\begin{array}{l}16.20 \\
15.40 \\
14.67 \\
14.26 \\
14.09 \\
14.43 \\
15.31 \\
15.27 \\
15.03 \\
15.19 \\
14.97 \\
15.09 \\
14.39 \\
14.49 \\
14.24 \\
13.97 \\
13.64 \\
13.36 \\
13.26 \\
15.78\end{array}$ & $\begin{array}{r}0.03 \\
5.16 \\
3.64 \\
6.69 \\
11.68 \\
14.94 \\
14.50 \\
19.69 \\
18.59 \\
10.12 \\
15.26 \\
14.22 \\
9.57 \\
9.30 \\
9.36 \\
8.53 \\
7.38 \\
6.21 \\
5.41 \\
8.25\end{array}$ & $\begin{array}{r}7.68 \\
8.38 \\
6.13 \\
13.48 \\
18.58 \\
23.60 \\
20.89 \\
24.48 \\
22.98 \\
12.49 \\
20.43 \\
19.46 \\
11.99 \\
10.40 \\
12.34 \\
10.93 \\
12.87 \\
8.38 \\
13.04 \\
12.07\end{array}$ & $\begin{array}{r}1644 . \\
1606 . \\
1643 . \\
1550 . \\
1620 . \\
1620 . \\
1520 . \\
1629 . \\
1324 . \\
9 . \\
2810 . \\
29 . \\
1239 . \\
1613 . \\
1+14 . \\
1037 . \\
1502 . \\
19 . \\
1654 . \\
1504 .\end{array}$ & $\begin{array}{r}3.04 \\
3.28 \\
-.23 \\
-1.20 \\
6.89 \\
4.78 \\
7.51 \\
8.91 \\
12.69 \\
8.47 \\
16.67 \\
9.35 \\
8.82 \\
8.12 \\
8.29 \\
4.51 \\
.91 \\
1.52 \\
-.06 \\
1.79\end{array}$ & $\begin{array}{r}2355 . \\
1455 . \\
252 . \\
603 . \\
2348 . \\
631 . \\
634 . \\
241 . \\
2400 . \\
720 . \\
20 . \\
2544 . \\
230 . \\
2334 . \\
29 . \\
2359 . \\
544 . \\
2400 . \\
629 . \\
147 .\end{array}$ & $\begin{array}{r}4.85 \\
3.92 \\
2.67 \\
3.70 \\
8.65 \\
11.21 \\
11.35 \\
14.73 \\
15.88 \\
9.85 \\
14.68 \\
13.52 \\
9.87 \\
9.00 \\
5.98 \\
7.75 \\
3.30 \\
5.85 \\
3.54 \\
5.66\end{array}$ \\
\hline
\end{tabular}

\begin{tabular}{|c|c|c|c|c|c|c|c|}
\hline DATE & $\begin{array}{c}\text { SURFACE } \\
\text { HATER }\end{array}$ & $\begin{array}{l}\text { DRY EULS } \\
\text { AIR }\end{array}$ & $\begin{array}{c}\text { MAXIYUM } \\
\text { AIR }\end{array}$ & $\begin{array}{l}\text { TIME OF } \\
\text { MAXIMUM }\end{array}$ & $\begin{array}{c}\text { MIXIMUM } \\
\text { AIR }\end{array}$ & $\begin{array}{l}\text { TIME of } \\
\text { MINIMUH }\end{array}$ & $\begin{array}{l}\text { WET BULB } \\
\text { AIR }\end{array}$ \\
\hline $\begin{array}{l}283 . \\
284 . \\
285 . \\
296 . \\
287 . \\
288 . \\
289 . \\
290 . \\
291 . \\
292 . \\
293 . \\
294 . \\
295 . \\
295 . \\
299 . \\
299 . \\
299 .\end{array}$ & $\begin{array}{l}12.42 \\
1=.16 \\
12.72 \\
11.54 \\
11.02 \\
10.63 \\
10.34 \\
10.15 \\
0.39 \\
9.07 \\
9.42 \\
9.36 \\
9.51 \\
9.25 \\
9.07 \\
8.84 \\
0.76\end{array}$ & $\begin{array}{l}9.46 \\
6.27 \\
1.58 \\
2.26 \\
4.35 \\
5.05 \\
5.34 \\
5.49 \\
3.94 \\
5.93 \\
6.30 \\
6.45 \\
7.76 \\
3.65 \\
5.60 \\
5.59 \\
7.06\end{array}$ & $\begin{array}{r}11.46 \\
11.72 \\
5.57 \\
3.46 \\
12.00 \\
6.38 \\
9.35 \\
10.49 \\
5.48 \\
7.53 \\
10.95 \\
9.95 \\
13.75 \\
7.36 \\
10.23 \\
12.95 \\
14.45\end{array}$ & 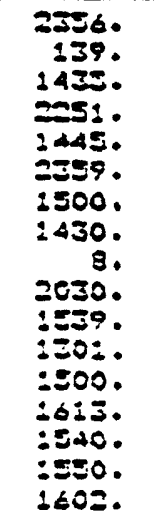 & $\begin{array}{r}7.08 \\
0.59 \\
-1.46 \\
.73 \\
.122 \\
3.81 \\
.32 \\
.47 \\
2.84 \\
3.79 \\
3.65 \\
3.72 \\
2.76 \\
.20 \\
2.43 \\
-.41 \\
.82\end{array}$ & $\begin{array}{r}1054 . \\
2838 . \\
825 . \\
779 . \\
709 . \\
657 . \\
2304 . \\
704 . \\
719 . \\
100 . \\
=347 . \\
: 00 . \\
=357 . \\
640 . \\
2400 . \\
647 . \\
553 .\end{array}$ & $\begin{array}{r}8.81 \\
4.43 \\
.67 \\
2.21 \\
2.90 \\
4.59 \\
4.01 \\
3.95 \\
2.40 \\
3.60 \\
=.43 \\
5.25 \\
6.85 \\
3.53 \\
=.05 \\
3.29 \\
4.52\end{array}$ \\
\hline
\end{tabular}


Table 3. Raft station: (1) Daily average wind speed at three levels above the lake surface; and (2) daily maximum and minimum wind speed at each level and the time they occurred.

[mph, miles per hour]

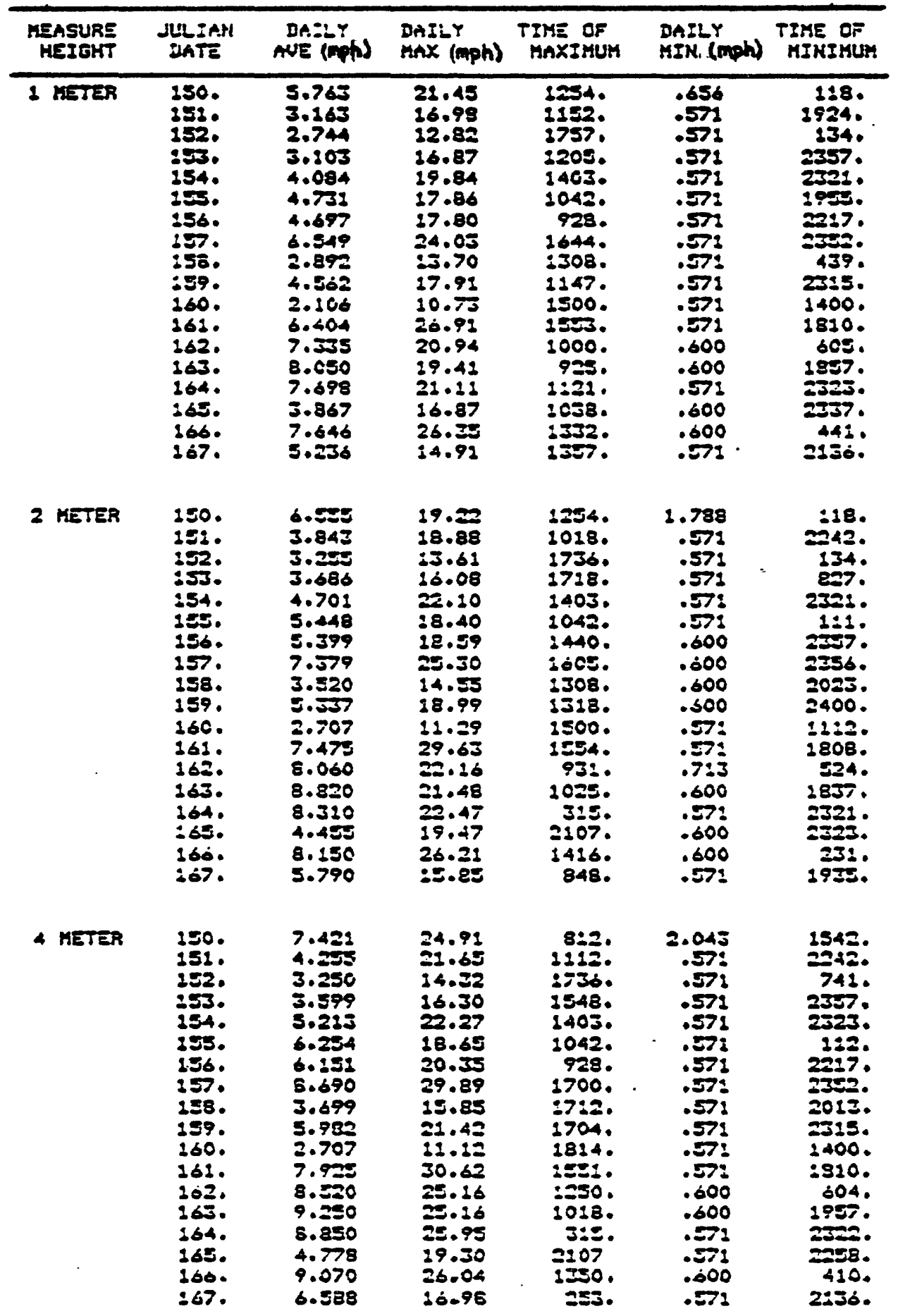


I'able 3. Raft station: (1) Daily average wind speed at three levels above the lake surface; and (2) daily maximum and minimum wind speed at each level and the time they occurred. [continued]

\begin{tabular}{|c|c|c|c|c|c|c|}
\hline $\begin{array}{l}\text { MEASURE } \\
\text { HEIGKT }\end{array}$ & $\begin{array}{l}\text { JULIAN } \\
\text { DATE }\end{array}$ & $\begin{array}{l}\text { DAIIY } \\
\text { AUERAEE }\end{array}$ & $\begin{array}{l}\text { DAILY } \\
\text { MAXIMUH }\end{array}$ & $\begin{array}{l}\text { TIME OF } \\
\text { MAXIMUM }\end{array}$ & $\begin{array}{l}\text { DAI:Y } \\
\text { MIKIMUM }\end{array}$ & $\begin{array}{l}\text { TIME OF } \\
\text { MINIMUH }\end{array}$ \\
\hline 2 VETEA & $\begin{array}{l}168 . \\
169 . \\
170 . \\
171 . \\
172 . \\
1730 \\
174 . \\
175 . \\
176 . \\
177 . \\
178 . \\
179 . \\
180 . \\
181 . \\
182 . \\
183 . \\
184 . \\
185 . \\
186 . \\
187 .\end{array}$ & $\begin{array}{l}1.772 \\
5.503 \\
6.959 \\
6.039 \\
5.571 \\
6.677 \\
1.585 \\
4.286 \\
7.461 \\
2.580 \\
4.180 \\
3.560 \\
5.5166 \\
5.604 \\
3.307 \\
4.953 \\
4.055 \\
7.890 \\
2.718 \\
6.356\end{array}$ & $\begin{array}{l}12.17 \\
20.24 \\
23.07 \\
18.17 \\
21.74 \\
29.58 \\
10.75 \\
14.38 \\
23.09 \\
14.94 \\
15.34 \\
13.81 \\
20.41 \\
16.33 \\
12.96 \\
20.38 \\
22.92 \\
29.87 \\
12.14 \\
23.35\end{array}$ & 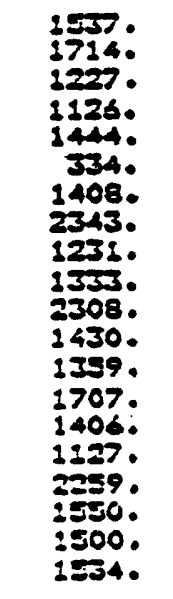 & $\begin{array}{l}.571 \\
.571 \\
.600 \\
.600 \\
.600 \\
.600 \\
.571 \\
.571 \\
.600 \\
.600 \\
.571 \\
.600 \\
.600 \\
.600 \\
.578 \\
.571 \\
.600 \\
.600 \\
.571 \\
.600\end{array}$ & $\begin{array}{r}2146 . \\
454 . \\
21: 5 . \\
23350 \\
2006 . \\
2400 . \\
2337 . \\
3530 \\
1859 . \\
2359 . \\
2127 . \\
2142 . \\
736 . \\
2400 . \\
2149 . \\
429 . \\
2042 . \\
2310 . \\
2057 . \\
417 .\end{array}$ \\
\hline I METER & $\begin{array}{l}168 . \\
169 . \\
170 . \\
17 ! . \\
172 . \\
1730 \\
174 . \\
138: \\
177 . \\
178 . \\
179 . \\
180 . \\
184 . \\
182 . \\
185 . \\
184 . \\
185 . \\
186 . \\
197 .\end{array}$ & $\begin{array}{l}2.527 \\
6.691 \\
8.320 \\
7.454 \\
6.642 \\
7.705 \\
2.080 \\
8.878 \\
3.137 \\
5.052 \\
4.528 \\
6.676 \\
6.232 \\
3.777 \\
3.981 \\
4.645 \\
8.660 \\
3.259 \\
6.996\end{array}$ & $\begin{array}{l}11.97 \\
21.74 \\
22.70 \\
18.99 \\
25.46 \\
19.70 \\
10.78 \\
12.95 \\
15.45 \\
16.30 \\
15.51 \\
20.26 \\
17.72 \\
12.35 \\
25.84 \\
26.38 \\
24.91 \\
11.80 \\
22.07\end{array}$ & $\begin{array}{l}1537 . \\
1253 . \\
1416 . \\
1137 . \\
1414 . \\
1455 . \\
1312 . \\
1317: \\
1535 . \\
2308 . \\
952 . \\
1359 . \\
1652 . \\
1406 . \\
1600 . \\
2140 . \\
2532 . \\
1524 . \\
1244 .\end{array}$ & $\begin{array}{r}.571 \\
.571 \\
2.184 \\
1.109 \\
.600 \\
.500 \\
.571 \\
2: \$ 99 \\
.600 \\
.571 \\
.600 \\
.656 \\
.600 \\
.578 \\
.578 \\
.600 \\
.911 \\
.600 \\
.600\end{array}$ & $\begin{array}{r}: 523 . \\
454 . \\
2059 . \\
2325 . \\
1124 . \\
2400 . \\
2337 . \\
1359: \\
2354 . \\
2127 . \\
643 . \\
326 . \\
2338 . \\
1152 . \\
435 . \\
2021 . \\
2=17 . \\
2306 . \\
305 .\end{array}$ \\
\hline 4 METER & $\begin{array}{l}168 . \\
169 . \\
170 . \\
171 . \\
170 . \\
173 . \\
1740 \\
175 . \\
176 . \\
177 . \\
178 . \\
179 . \\
180 . \\
18: \\
182 . \\
183 . \\
184 . \\
195 . \\
186 . \\
387 .\end{array}$ & $\begin{array}{l}2.116 \\
7.159 \\
8.990 \\
8.145 \\
7.089 \\
8.590 \\
1.778 \\
5.247 \\
8.350 \\
3.116 \\
5.447 \\
4.827 \\
7.470 \\
6.720 \\
3.965 \\
6.445 \\
4.772 \\
9.750 \\
2.965 \\
7.272\end{array}$ & $\begin{array}{l}13.16 \\
21.28 \\
29.41 \\
20.43 \\
23.38 \\
21.40 \\
10.44 \\
28.99 \\
22.73 \\
14.80 \\
16.19 \\
17.24 \\
18.14 \\
19.11 \\
13.09 \\
28.05 \\
28.64 \\
27.23 \\
12.74 \\
24.23\end{array}$ & $\begin{array}{r}1557 . \\
1253 . \\
1410 . \\
1226 . \\
1414 . \\
1455 . \\
1312 . \\
542 . \\
1211 . \\
1334 . \\
1307 . \\
952 . \\
: 359 . \\
1624 . \\
1406 . \\
1600 . \\
2140 . \\
1520 . \\
1524 . \\
1509 .\end{array}$ & $\begin{array}{r}.571 \\
.571 \\
1.930 \\
.571 \\
.571 \\
.571 \\
.571 \\
.571 \\
.600 \\
.571 \\
.571 \\
.571 \\
.600 \\
.500 \\
.571 \\
.571 \\
.571 \\
.600 \\
.571 \\
.600\end{array}$ & $\begin{array}{r}2349 . \\
454 . \\
545 . \\
2325 . \\
705 . \\
2143 . \\
2357 . \\
1210 . \\
2011 . \\
2142 . \\
2127 . \\
313 . \\
726 . \\
2400 . \\
2119 . \\
429 . \\
1405 . \\
2403 . \\
2515 . \\
413 .\end{array}$ \\
\hline
\end{tabular}


Table 3. Raft station: (1) Daily average wind speed at three levels above the lake surface; and (2) daily maximum and minimum wind speed at each level and the time they occurred. [continued]

\begin{tabular}{|c|c|c|c|c|c|c|}
\hline $\begin{array}{l}\text { MEASURE } \\
\text { HEIEHT }\end{array}$ & $\begin{array}{l}\text { JUIAN } \\
\text { DATE }\end{array}$ & $\begin{array}{l}\text { DAILY } \\
\text { AVERAGE }\end{array}$ & $\begin{array}{l}\text { DAILY } \\
\text { MAXIMUM }\end{array}$ & $\begin{array}{l}\text { TIME OF } \\
\text { HAXIMUK }\end{array}$ & $\begin{array}{l}\text { DAZLY } \\
\text { MINIMUM }\end{array}$ & $\begin{array}{l}\text { TIME OF } \\
\text { MINIMUM }\end{array}$ \\
\hline I HETER & $\begin{array}{l}188 . \\
289 . \\
290 . \\
191 . \\
192 . \\
293 . \\
194 . \\
195 . \\
196 . \\
197 . \\
198 . \\
199 . \\
200 .\end{array}$ & $\begin{array}{l}7.757 \\
2.394 \\
6.173 \\
7.636 \\
7.890 \\
4.863 \\
7.237 \\
5.214 . \\
6.149 \\
4.384 \\
1.950 \\
2.889 \\
2.169\end{array}$ & $\begin{array}{l}24.96 \\
13.98 \\
16.92 \\
22.39 \\
24.68 \\
15.74 \\
21.82 \\
25.62 \\
20.89 \\
15.54 \\
13.22 \\
13.75 \\
12.42\end{array}$ & $\begin{array}{l}1532 . \\
2312 . \\
2215 . \\
1102 . \\
1118 . \\
1306 . \\
1421 . \\
16130 \\
1449 . \\
1308 . \\
1509 . \\
155 . \\
1457 .\end{array}$ & $\begin{array}{r}1.901 \\
.571 \\
1.477 \\
.600 \\
.600 \\
.600 \\
.600 \\
.600 \\
1.194 \\
.571 \\
.571 \\
.571 \\
.571\end{array}$ & $\begin{array}{r}231 . \\
1938 . \\
745 . \\
2353 . \\
2400 . \\
723 . \\
2953 . \\
225 . \\
323 . \\
2058 . \\
2138 . \\
2135 . \\
2200 .\end{array}$ \\
\hline 2 METER & $\begin{array}{l}189 . \\
189 . \\
190 . \\
190^{\circ} \\
192 . \\
193 . \\
194 . \\
190^{\circ} \\
196 . \\
197 . \\
198 . \\
199 . \\
200 .\end{array}$ & $\begin{array}{l}8.320 \\
3.307 \\
7.517 \\
8.490 \\
8.420 \\
5.420 \\
7.970 \\
6.068 \\
7.487 \\
4.873 \\
2.545 \\
3.643 \\
2.789\end{array}$ & $\begin{array}{l}28.02 \\
14.18 \\
18.14 \\
21.79 \\
20.52 \\
16.53 \\
21.13 \\
16.41 \\
20.80 \\
16.90 \\
13.84 \\
13.73 \\
13.92\end{array}$ & $\begin{array}{r}: 532 . \\
2557 . \\
1212 . \\
1252 . \\
1138 . \\
1306 . \\
1343 . \\
1401 . \\
1449 . \\
15 . \\
1509 . \\
556 . \\
1454 .\end{array}$ & $\begin{array}{r}2.892 \\
.600 \\
2.213 \\
1.222 \\
.600 \\
.600 \\
1.930 \\
1.535 \\
1.986 \\
.571 \\
.571 \\
.600 \\
.571\end{array}$ & $\begin{array}{r}2524 . \\
2032 . \\
753 . \\
2353 . \\
2400 . \\
609 . \\
1959 . \\
2234 . \\
323 . \\
2346 . \\
2158 . \\
2400 . \\
2200 .\end{array}$ \\
\hline 4 METER & $\begin{array}{l}188 . \\
189 . \\
190 . \\
191 . \\
192 . \\
195 . \\
194 . \\
195 . \\
196 . \\
197 . \\
193 . \\
199 . \\
200 .\end{array}$ & $\begin{array}{l}8.650 \\
3.298 \\
8.310 \\
9.230 \\
9.010 \\
5.307 \\
8.420 \\
6.366 \\
8.070 \\
4.962 \\
2.379 \\
3.737 \\
2.783\end{array}$ & $\begin{array}{l}25.02 \\
36.33 \\
17.66 \\
22.95 \\
28.05 \\
17.12 \\
23.04 \\
17.38 \\
21.25 \\
18.25 \\
13.61 \\
15.88 \\
15.28\end{array}$ & $\begin{array}{l}1542 . \\
2214 . \\
1215 . \\
1112 . \\
1405 . \\
1253 . \\
1453 . \\
1502 . \\
1506 . \\
106 . \\
1509 . \\
1053 . \\
1457 .\end{array}$ & $\begin{array}{r}1.031 \\
.571 \\
2.609 \\
.600 \\
.600 \\
.600 \\
.600 \\
.600 \\
.628 \\
.571 \\
.571 \\
.571 \\
.571\end{array}$ & $\begin{array}{r}2324 . \\
754 . \\
356 . \\
2400 . \\
2400 . \\
800 . \\
1959 . \\
2933 . \\
325 . \\
2346 . \\
2158 . \\
2135 . \\
2200 .\end{array}$ \\
\hline
\end{tabular}


Table 3. Raft station: (1) Daily average wind speed at three levels above the lake surface; and (2) daily maximum and minimum wind speed at each level and the time they occurred. [continued]

\begin{tabular}{|c|c|c|c|c|c|c|c|}
\hline \multicolumn{2}{|c|}{$\begin{array}{l}\text { MEASURE } \\
\text { HEIEHT }\end{array}$} & $\begin{array}{l}\text { JULIAN } \\
\text { DATE }\end{array}$ & $\begin{array}{l}\text { DAILY } \\
\text { AUEFHEE }\end{array}$ & $\begin{array}{l}\text { DAI:-Y } \\
\text { MAXIMUH }\end{array}$ & $\begin{array}{l}\text { TIME OF } \\
\text { MAXIMUY }\end{array}$ & $\begin{array}{l}\text { DAILY } \\
\text { MIRIHUM }\end{array}$ & $\begin{array}{l}\text { TIME OF } \\
\text { MINIKUM }\end{array}$ \\
\hline & $\begin{array}{l}\text { METER } \\
\text { - }\end{array}$ & $\begin{array}{l}201 . \\
202 . \\
203 . \\
204 . \\
205 . \\
206 . \\
207 . \\
208 . \\
209 . \\
210 . \\
212 . \\
212 . \\
213 . \\
214 . \\
215 . \\
216 .\end{array}$ & $\begin{array}{l}3.065 \\
2.984 \\
3.359 \\
4.260 \\
1.800 \\
1.903 \\
5.094 \\
3.449 \\
2.307 \\
2.573 \\
2.257 \\
4.763 \\
1.552 \\
3.642 \\
2.608 \\
2.553\end{array}$ & $\begin{array}{r}14.26 \\
10.27 \\
15.82 \\
20.09 \\
8.75 \\
10.67 \\
20.77 \\
14.22 \\
14.11 \\
21.94 \\
15.71 \\
17.32 \\
6.91 \\
17.21 \\
12.59 \\
9.35\end{array}$ & $\begin{array}{r}1228 . \\
518 . \\
1612 . \\
2253 . \\
1156 . \\
2200 . \\
12140 \\
759 . \\
1700 . \\
1348 . \\
549 . \\
1401 . \\
1141 . \\
910 . \\
1255 . \\
1258 .\end{array}$ & $\begin{array}{r}.571 \\
.571 \\
.571 \\
.571 \\
.571 \\
.600 \\
.600 \\
.600 \\
.571 \\
.571 \\
.571 \\
.600 \\
.571 \\
.500 \\
.571 \\
.571\end{array}$ & $\begin{array}{r}1924 . \\
139 . \\
1934 . \\
500 . \\
2013 . \\
2356 . \\
500 . \\
2351 . \\
2202 . \\
2130 . \\
2005 . \\
2400 . \\
1829 . \\
2347 . \\
2305 . \\
312 .\end{array}$ \\
\hline 2 & METES & $\begin{array}{l}201 . \\
202 . \\
203 . \\
204 . \\
205 . \\
206 . \\
207 . \\
208 . \\
209 . \\
210 . \\
211 . \\
212 . \\
213 . \\
214 . \\
215 . \\
216 .\end{array}$ & $\begin{array}{l}3.726 \\
3.771 \\
3.524 \\
4.897 \\
2.397 \\
2.530 \\
6.204 \\
6.194 \\
2.677 \\
2.775 \\
2.891 \\
3.702 \\
2.142 \\
4.539 \\
3.023 \\
3.404\end{array}$ & $\begin{array}{l}16.37 \\
10.56 \\
16.19 \\
19.10 \\
9.96 \\
12.45 \\
19.50 \\
14.35 \\
13.84 \\
12.79 \\
16.70 \\
19.95 \\
7.76 \\
15.12 \\
12.85 \\
10.19\end{array}$ & $\begin{array}{l}1921 . \\
518 . \\
1612 . \\
1311 . \\
1 \pm 56 . \\
1351 . \\
1214 . \\
919 . \\
1700 . \\
1256 . \\
549 . \\
1046 . \\
1423 . \\
910 . \\
1252 . \\
1715 .\end{array}$ & $\begin{array}{r}.571 \\
.571 \\
.571 \\
.571 \\
.600 \\
.571 \\
.600 \\
1.166 \\
.571 \\
.571 \\
.571 \\
.600 \\
.600 \\
.600 \\
.571 \\
.571\end{array}$ & $\begin{array}{r}114 . \\
\vdots 39 . \\
2135 . \\
31 . \\
2359 . \\
850 . \\
351 . \\
3351 . \\
332 . \\
-201 . \\
19 . \\
2400 . \\
2225 . \\
2344 . \\
932 . \\
51 .\end{array}$ \\
\hline 4 & METER & $\begin{array}{l}201 . \\
202 . \\
205 . \\
204 . \\
205 . \\
206 . \\
207 . \\
208 . \\
209 . \\
210 . \\
211 . \\
212 . \\
213 . \\
214 . \\
215 . \\
216 .\end{array}$ & $\begin{array}{l}3.278 \\
3.936 \\
3.945 \\
5.381 \\
1.964 \\
3.396 \\
6.453 \\
6.518 \\
2.396 \\
3.861 \\
2.712 \\
6.427 \\
1.846 \\
4.900 \\
3.121 \\
3.444\end{array}$ & $\begin{array}{r}22.70 \\
13.13 \\
16.58 \\
24.08 \\
10.44 \\
12.77 \\
18.74 \\
14.55 \\
14.29 \\
13.73 \\
15.74 \\
21.88 \\
8.69 \\
12.01 \\
15.37 \\
10.08\end{array}$ & $\begin{array}{r}944 . \\
9518 . \\
1656 . \\
1352 . \\
1235 . \\
1426 . \\
1441 . \\
909 . \\
1724 . \\
1346 . \\
1549 . \\
1046 . \\
1141 . \\
910 . \\
1233 . \\
1733 .\end{array}$ & $\begin{array}{l}.571 \\
.571 \\
.571 \\
.571 \\
.571 \\
.571 \\
.600 \\
.600 \\
.571 \\
.571 \\
.571 \\
.571 \\
.571 \\
.600 \\
.571 \\
.571\end{array}$ & $\begin{array}{r}1926 . \\
130 . \\
2135 . \\
2156 . \\
2152 . \\
2050 . \\
2927 . \\
2353 . \\
2202 . \\
2130 . \\
2005 . \\
2106 . \\
1829 . \\
2345 . \\
2303 . \\
621 .\end{array}$ \\
\hline
\end{tabular}


Table 3. Raft station: (1) Daily average wind speed at three levels above the lake surface; and (2) daily maximum and minimum wind speed at each level and the time they occurred. [continued]

\begin{tabular}{|c|c|c|c|c|c|c|}
\hline $\begin{array}{l}\text { MEASURE } \\
\text { HEIBHT }\end{array}$ & $\begin{array}{l}\text { JULIAN } \\
\text { DATE }\end{array}$ & $\begin{array}{l}\text { DAILY } \\
\text { AUERAGE }\end{array}$ & $\begin{array}{l}\text { DAILY } \\
\text { MAXIYUM }\end{array}$ & $\begin{array}{l}\text { TIME of } \\
\text { MAXIMUH }\end{array}$ & $\begin{array}{l}\text { BAILY } \\
\text { MINIMUY }\end{array}$ & $\begin{array}{l}\text { PIME OF } \\
\text { MINIMUK }\end{array}$ \\
\hline 1 METER & $\begin{array}{l}217 . \\
218 . \\
219 . \\
220 . \\
221 . \\
220 . \\
223 . \\
224 . \\
2=5 . \\
226 . \\
227 . \\
228 . \\
229 . \\
230 .\end{array}$ & $\begin{array}{l}3.635 \\
1.777 \\
7.075 \\
3.524 \\
3.000 \\
4.672 \\
2.311 \\
5.974 \\
4.609 \\
1.881 \\
2.678 \\
1.926 \\
1.687 \\
4.186\end{array}$ & $\begin{array}{r}13.25 \\
7.76 \\
23.04 \\
15.91 \\
22.03 \\
13.25 \\
12.00 \\
17.09 \\
10.04 \\
10.36 \\
14.18 \\
16.27 \\
9.23 \\
15.00\end{array}$ & $\begin{array}{l}1233 . \\
1305 . \\
1105 . \\
1202 . \\
1904 . \\
1247 . \\
811 . \\
1319 . \\
1442 . \\
1448 . \\
1218 . \\
1516 . \\
1420 . \\
1320 .\end{array}$ & $\begin{array}{r}.571 \\
.571 \\
.600 \\
.600 \\
.571 \\
.600 \\
.571 \\
.600 \\
.571 \\
.571 \\
.600 \\
.571 \\
.600 \\
.571\end{array}$ & $\begin{array}{r}1946 . \\
6260 \\
2400 . \\
2400 . \\
1641 . \\
2350 . \\
40 . \\
917 . \\
2307 . \\
1911 . \\
2400 . \\
2920 . \\
2357 . \\
251 .\end{array}$ \\
\hline 2 METER & $\begin{array}{l}217 . \\
218 . \\
219 . \\
220 . \\
221 . \\
229 . \\
223 . \\
224 . \\
225 . \\
226 . \\
227 . \\
2=18 . \\
229 . \\
230 .\end{array}$ & $\begin{array}{l}4.177 \\
2.347 \\
7.796 \\
4.207 \\
3.762 \\
5.529 \\
2.966 \\
7.005 \\
5.054 \\
2.313 \\
3.276 \\
2.491 \\
2.164 \\
3.095\end{array}$ & $\begin{array}{r}13.47 \\
8.60 \\
24.45 \\
19.36 \\
12.93 \\
16.05 \\
11.92 \\
19.50 \\
17.18 \\
11.53 \\
15.31 \\
17.97 \\
8.92 \\
16.10\end{array}$ & $\begin{array}{l}1253 . \\
949 . \\
12130 \\
1119 . \\
1904 . \\
1201 . \\
811 . \\
1336 . \\
1442 . \\
1249 . \\
1218 . \\
1516 . \\
1420 . \\
1416 .\end{array}$ & $\begin{array}{l}.571 \\
.571 \\
.600 \\
.571 \\
.571 \\
.600 \\
.600 \\
.628 \\
.571 \\
.571 \\
.571 \\
.571 \\
.022 \\
.571\end{array}$ & $\begin{array}{l}2546 . \\
1650 . \\
2354 . \\
2129 . \\
1639 . \\
2925 . \\
2139 . \\
29 . \\
3307 . \\
2911 . \\
2305 . \\
2920 . \\
1727 . \\
251 .\end{array}$ \\
\hline 4 METEN & $\begin{array}{l}217 . \\
218 . \\
219 . \\
220 . \\
221 . \\
222 . \\
223 . \\
224 . \\
225 . \\
226 . \\
227 . \\
228 . \\
229 . \\
230 .\end{array}$ & $\begin{array}{l}4.078 \\
2.068 \\
8.300 \\
4.420 \\
3.796 \\
3.990 \\
2.803 \\
7.349 \\
5.484 . \\
2.238 \\
3.068 \\
2.273 \\
\vdots .640 \\
5.273\end{array}$ & $\begin{array}{r}13.39 \\
9.63 \\
27.14 \\
19.61 \\
12.96 \\
17.69 \\
13.08 \\
21.42 \\
17.38 \\
10.84 \\
14.41 \\
18.03 \\
8.75 \\
17.97\end{array}$ & $\begin{array}{l}1015 . \\
949 . \\
1355 . \\
2111 . \\
1840 . \\
1611 . \\
821 . \\
1319 . \\
1442 . \\
1515 . \\
1033 . \\
1516 . \\
1422 . \\
1342 .\end{array}$ & $\begin{array}{l}.571 \\
.571 \\
.600 \\
.571 \\
.571 \\
.600 \\
.571 \\
.600 \\
.571 \\
.571 \\
.571 \\
.545 \\
.571 \\
.571\end{array}$ & $\begin{array}{l}2146 . \\
2019 . \\
2400 . \\
2229 . \\
1641 . \\
2345 . \\
2103 . \\
1043 . \\
2307 . \\
2055 . \\
2305 . \\
623 . \\
1958 . \\
258 .\end{array}$ \\
\hline
\end{tabular}


Table 3. Raft station: (1) Daily average wind speed at three levels above the lake surface; and (2) daily maximum and minimum wind speed at each level and the time they occurred. [continued]

\begin{tabular}{|c|c|c|c|c|c|c|}
\hline $\begin{array}{l}\text { MEASURE } \\
\text { HETEHT }\end{array}$ & DUIAN & $\begin{array}{c}\text { DAILY } \\
\text { AUEFAGE }\end{array}$ & $\begin{array}{l}\text { DAILY } \\
\text { MAXIHLM }\end{array}$ & $\begin{array}{l}\text { TIME OF } \\
\text { MAXIHUM }\end{array}$ & $\begin{array}{l}\text { DAILY } \\
\text { HINIAUH }\end{array}$ & $\begin{array}{l}\text { TIME OF } \\
\text { MINIMUN }\end{array}$ \\
\hline 1 MITER & $\begin{array}{l}233 . \\
232 . \\
233 . \\
234 . \\
235 . \\
236 . \\
237 . \\
238 . \\
239 . \\
240 . \\
241 . \\
242 . \\
245 . \\
244 .\end{array}$ & $\begin{array}{l}3.490 \\
3.448 \\
4.449 \\
1.922 \\
4.286 \\
4.344 \\
4.026 \\
2.971 \\
2.373 \\
4.872 \\
3.552 \\
1.973 \\
3.737 \\
4.753\end{array}$ & $\begin{array}{l}19.19 \\
14.38 \\
18.72 \\
11.24 \\
18.11 \\
13.70 \\
11.63 \\
25.87 \\
14.07 \\
15.31 \\
24.34 \\
10.87 \\
16.53 \\
16.19\end{array}$ & $\begin{array}{r}851 . \\
1630 . \\
1636 . \\
1130 . \\
1313 . \\
209 . \\
1230 . \\
29 . \\
: 910 . \\
2007 . \\
800 . \\
1049 . \\
1220 . \\
: 329 .\end{array}$ & $\begin{array}{l}.600 \\
.571 \\
.600 \\
.571 \\
.571 \\
.571 \\
.600 \\
.571 \\
.600 \\
.600 \\
.571 \\
.571 \\
.600 \\
.600\end{array}$ & $\begin{array}{r}2400 . \\
632 . \\
2400 . \\
2022 . \\
101 . \\
2039 . \\
2134 . \\
1916 . \\
2308 . \\
2400 . \\
2245 . \\
2245 . \\
2306 . \\
1527 .\end{array}$ \\
\hline 2 METER & $\begin{array}{l}231 . \\
232 . \\
233 . \\
234 . \\
235 . \\
236 . \\
236 . \\
238 . \\
239 . \\
240 . \\
241 . \\
242 . \\
243 . \\
244 .\end{array}$ & $\begin{array}{l}0.245 \\
4.279 \\
5.334 \\
2.486 \\
5.409 \\
5.334 \\
4.766 \\
3.487 \\
2.907 \\
5.385 \\
4.263 \\
2.485 \\
4.477 \\
5.366\end{array}$ & $\begin{array}{l}29.44 \\
16.30 \\
17.24 \\
12.85 \\
18.89 \\
14.46 \\
13.81 \\
28.47 \\
13.33 \\
16.22 \\
24.76 \\
12.37 \\
17.18 \\
16.13\end{array}$ & $\begin{array}{r}254 . \\
1724 . \\
1649 . \\
1215 . \\
1313 . \\
1021 . \\
1122 . \\
10 . \\
1910 . \\
2005 . \\
432 . \\
1245 . \\
1220 . \\
1528 .\end{array}$ & $\begin{array}{l}.600 \\
.571 \\
.600 \\
.571 \\
.571 \\
.600 \\
.571 \\
.571 \\
.600 \\
.600 \\
.571 \\
.571 \\
.000 \\
.600\end{array}$ & $\begin{array}{r}2400 . \\
2027 . \\
2400 . \\
2023 . \\
101 . \\
2341 . \\
1502 . \\
2128 . \\
2308 . \\
2400 . \\
2247 . \\
2245 . \\
1831 . \\
727 .\end{array}$ \\
\hline 4 METER & $\begin{array}{l}231 . \\
232 . \\
233 . \\
234 . \\
235 . \\
236 . \\
237 . \\
238 . \\
239 . \\
240 . \\
241 . \\
243 . \\
243 . \\
244 .\end{array}$ & $\begin{array}{l}7.031 \\
4.581 \\
3.914 \\
2.276 \\
3.879 \\
5.568 \\
4.789 \\
3.634 \\
3.054 \\
6.141 \\
4.502 \\
2.290 \\
4.840 \\
6.221\end{array}$ & $\begin{array}{l}24.37 \\
16.84 \\
18.14 \\
15.39 \\
19.29 \\
17.69 \\
16.36 \\
30.51 \\
15.38 \\
21.17 \\
29.12 \\
12.31 \\
17.29 \\
18.06\end{array}$ & $\begin{array}{r}829 . \\
1724 . \\
1636 . \\
1343 . \\
1314 . \\
1621 . \\
322 . \\
8 . \\
1910 . \\
3014 . \\
437 . \\
1524 . \\
1305 . \\
1048 .\end{array}$ & $\begin{array}{l}.571 \\
.571 \\
.600 \\
.571 \\
.571 \\
.600 \\
.571 \\
.571 \\
.317 \\
.600 \\
.571 \\
.571 \\
.600 \\
.600\end{array}$ & $\begin{array}{l}2142 . \\
1044 . \\
2400 . \\
2025 . \\
103 . \\
2400 . \\
1505 . \\
1916 . \\
752 . \\
2400 . \\
2247 . \\
2945 . \\
2020 . \\
2357 .\end{array}$ \\
\hline
\end{tabular}


Table 3. Raft station: (1) Daily average wind speed at three levels above the lake surface; and (2) daily maximum and minimum wind speed at each level and the time they occurred. [continued]

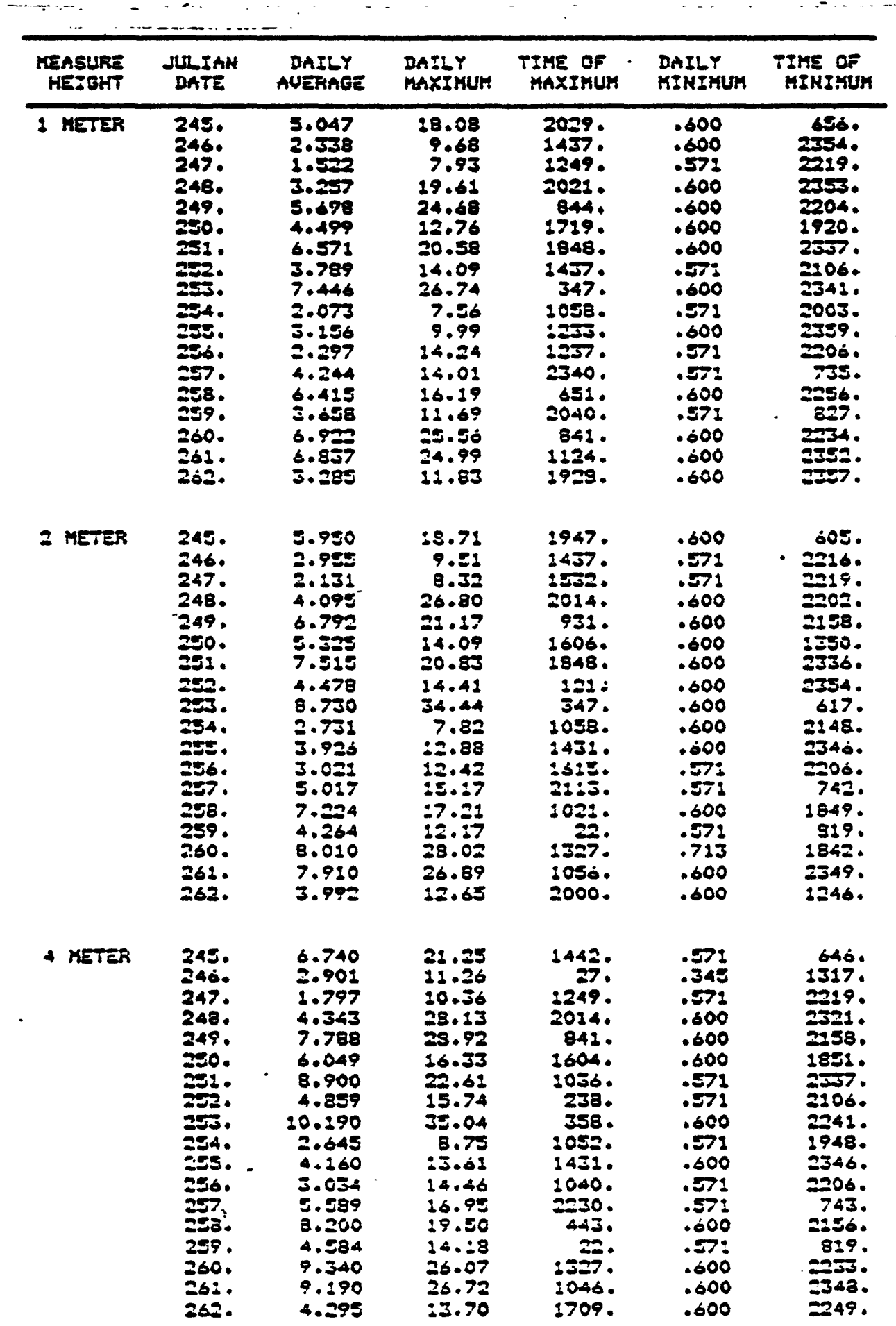


Table 3. Raft station: (1) Daily average wind speed at three levels above the lake surface; and (2) daily maximum and minimum wind speed at each level and the time they occurred. [continued]

\begin{tabular}{|c|c|c|c|c|c|c|}
\hline $\begin{array}{l}\text { MEASURE } \\
\text { HEISHT }\end{array}$ & $\begin{array}{l}\text { ULIAN } \\
\text { DATE }\end{array}$ & $\begin{array}{l}\text { IAIILY } \\
\text { AVERAEE }\end{array}$ & $\begin{array}{l}\text { BAË:Y } \\
\text { MAXIHUA }\end{array}$ & $\begin{array}{l}\text { Time of } \\
\text { HAXIKUM }\end{array}$ & $\begin{array}{l}\text { DAILY } \\
\text { MINIHUM }\end{array}$ & $\begin{array}{l}\text { TIME OF } \\
\text { MINIMUM }\end{array}$ \\
\hline 1 METER & $\begin{array}{l}265 . \\
2640 \\
2650 \\
2660 \\
2670 \\
2680 \\
269 . \\
2700 \\
2710 \\
270 . \\
2750 \\
2740 \\
2750 \\
276 . \\
277 . \\
278 . \\
279 . \\
290 . \\
281 . \\
382 .\end{array}$ & $\begin{array}{l}7.579 \\
7.613 \\
6.349 \\
4.696 \\
5.600 \\
2.773 \\
1.368 \\
3.5292 \\
6.386 \\
3.955 \\
3.294 \\
4.098 \\
2.920 \\
4.268 \\
2.406 \\
6.299 \\
2.972 \\
3.593 \\
1.661 \\
8.020\end{array}$ & $\begin{array}{l}21.03 \\
27.39 \\
18.85 \\
16.84 \\
19.30 \\
22.14 \\
10.39 \\
19.70 \\
18.76 \\
14.04 \\
13.10 \\
12.31 \\
12.65 \\
13.25 \\
9.08 \\
20.86 \\
15.03 \\
13.92 \\
10.50 \\
24.20\end{array}$ & $\begin{array}{r}1701 . \\
1358 . \\
317 . \\
1339 . \\
128 . \\
1312 . \\
1236 . \\
2357 . \\
446 . \\
526 . \\
1440 . \\
353 . \\
1440 . \\
106 . \\
1358 . \\
2029 . \\
1028 . \\
1431 . \\
1512 . \\
1530 .\end{array}$ & $\begin{array}{r}.911 \\
1.958 \\
1600 \\
.571 \\
.571 \\
.571 \\
.571 \\
.571 \\
.600 \\
.600 \\
.600 \\
.600 \\
.600 \\
.600 \\
.571 \\
.600 \\
.571 \\
.600 \\
.571 \\
.571\end{array}$ & $\begin{array}{r}26 . \\
1306 . \\
2400 . \\
304 . \\
2307 . \\
734 . \\
2011 . \\
439 . \\
1511 . \\
2347 . \\
1342 . \\
2400 . \\
1753 . \\
2339 . \\
1124 . \\
420 . \\
2530 \\
2400 . \\
1859 . \\
25 .\end{array}$ \\
\hline I METER & $\begin{array}{l}263 . \\
264 . \\
265 . \\
266 . \\
267 . \\
268 . \\
269 . \\
270 . \\
271 . \\
272 . \\
273 . \\
274 . \\
275 . \\
276 . \\
277 . \\
275 . \\
279 . \\
250 . \\
281 . \\
282 .\end{array}$ & $\begin{array}{l}8.600 \\
8.590 \\
7.392 \\
5.704 \\
6.555 \\
3.366 \\
1.759 \\
6.249 \\
7.647 \\
4.656 \\
3.988 \\
4.927 \\
3.593 \\
5.036 \\
3.146 \\
7.191 \\
3.888 \\
4.278 \\
2.403 \\
9.390\end{array}$ & $\begin{array}{r}23.79 \\
20.97 \\
20.41 \\
19.36 \\
29.81 \\
14.12 \\
9.74 \\
19.27 \\
19.84 \\
16.95 \\
13.30 \\
13.92 \\
14.38 \\
13.73 \\
13.33 \\
21.51 \\
14.89 \\
14.43 \\
10.67 \\
27.90\end{array}$ & $\begin{array}{r}1701 . \\
1134 . \\
757 . \\
1514 . \\
134 . \\
1338 . \\
1336 . \\
12350 . \\
2357 . \\
6330 \\
526 . \\
2328 . \\
353 . \\
1438 . \\
399 . \\
1308 . \\
2018 . \\
1300 . \\
1445 . \\
1446 . \\
1014 .\end{array}$ & $\begin{array}{r}1.739 \\
3.005 \\
.571 \\
.571 \\
.571 \\
.571 \\
.571 \\
.571 \\
.600 \\
.600 \\
.600 \\
.600 \\
.600 \\
.600 \\
.571 \\
.600 \\
.600 \\
.600 \\
.571 \\
.600\end{array}$ & 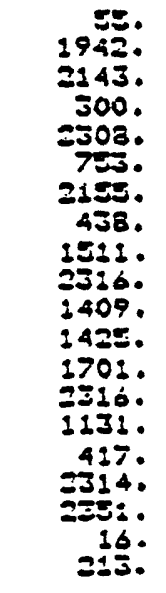 \\
\hline 4 METER & $\begin{array}{l}263 . \\
264 . \\
265 . \\
266 . \\
26 \% . \\
268 . \\
269 . \\
270 . \\
271 . \\
272 . \\
273 . \\
271 . \\
275 . \\
270 . \\
277 . \\
278 . \\
279 . \\
220 . \\
201 . \\
262 .\end{array}$ & $\begin{array}{r}10.240 \\
10.130 \\
8.700 \\
6.532 \\
7.472 \\
3.960 \\
1.670 \\
7.425 \\
8.980 \\
4.995 \\
4.404 \\
5.553 \\
3.708 \\
5.310 \\
3.249 \\
8.450 \\
4.1211 \\
4.502 \\
=.350 \\
10.560\end{array}$ & $\begin{array}{l}23.89 \\
22.47 \\
22.13 \\
20.80 \\
21.99 \\
15.45 \\
20.25 \\
23.89 \\
22.39 \\
2 \pm .71 \\
10.36 \\
15.00 \\
19.74 \\
15.17 \\
14.04 \\
25.67 \\
18.38 \\
15.05 \\
12.05 \\
26.26\end{array}$ & 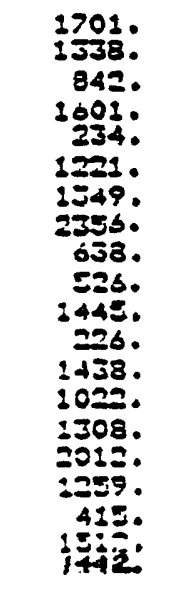 & $\begin{array}{l}.650 \\
3.147 \\
.600 \\
.571 \\
.571 \\
.571 \\
.571 \\
.571 \\
.600 \\
.600 \\
.573 \\
.600 \\
.600 \\
.600 \\
.578 \\
.600 \\
.572 \\
.600 \\
.671 \\
.571\end{array}$ & 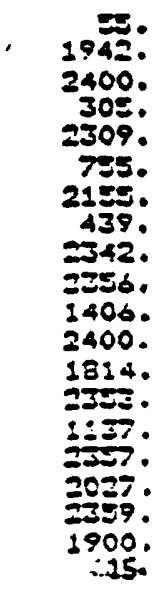 \\
\hline
\end{tabular}


Table 3. Raft station: (1) Daily average wind speed at three levels above the lake surface; and (2) daily maximum and minimum wind speed at each level and the time they occurred. [continued]

\begin{tabular}{|c|c|c|c|c|c|c|c|}
\hline \multicolumn{2}{|c|}{$\begin{array}{l}\text { MEASURE } \\
\text { HEIEHT }\end{array}$} & $\begin{array}{l}\text { JLIAN } \\
\text { DATE }\end{array}$ & $\begin{array}{l}\text { DAILY } \\
\text { AUERAGE }\end{array}$ & $\begin{array}{l}\text { DAILY } \\
\text { nAXIHUH }\end{array}$ & $\begin{array}{l}\text { TIME OF } \\
\text { HRXIMUH }\end{array}$ & $\begin{array}{l}\text { DAILY } \\
\text { MINIMUK }\end{array}$ & $\begin{array}{l}\text { TIME OF } \\
\text { MINIAUY }\end{array}$ \\
\hline & METER & $\begin{array}{l}383 . \\
284 . \\
285 . \\
286 . \\
287 . \\
288 . \\
289 . \\
390 . \\
291 . \\
292 . \\
293 . \\
294 . \\
295 . \\
296 . \\
397 . \\
299 . \\
299 .\end{array}$ & $\begin{array}{l}7.194 \\
3.999 \\
4.536 \\
4.281 \\
3.717 \\
5.751 \\
3.741 \\
2.972 \\
4.778 \\
3.831 \\
4.335 \\
2.451 \\
.949 \\
3.611 \\
4.095 \\
3.247 \\
2.560\end{array}$ & $\begin{array}{l}25.26 \\
18.14 \\
15.34 \\
11.39 \\
12.43 \\
18.85 \\
18.14 \\
18.03 \\
13.68 \\
14.24 \\
14.18 \\
10.16 \\
6.01 \\
11.03 \\
14.03 \\
29.26 \\
12.45\end{array}$ & 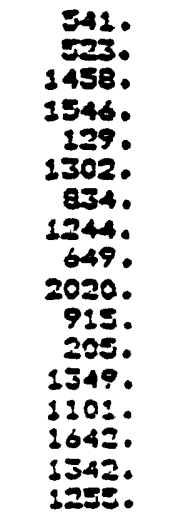 & $\begin{array}{r}.600 \\
.600 \\
.600 \\
.571 \\
.571 \\
.600 \\
.571 \\
.600 \\
.600 \\
1.449 \\
.571 \\
.571 \\
.571 \\
.571 \\
.571 \\
.579 \\
.571\end{array}$ & $\begin{array}{l}2342 . \\
2324 . \\
2229 . \\
1748 . \\
2954 . \\
2400 . \\
2307 . \\
2345 . \\
2241 . \\
1740 \\
1759 \\
1209 . \\
21140 \\
224 . \\
23150 \\
2329 . \\
1627 .\end{array}$ \\
\hline 2 & METER & $\begin{array}{l}283 . \\
284 . \\
295 . \\
286 . \\
287 . \\
288 . \\
289 . \\
290 . \\
291 . \\
292 . \\
293 . \\
294 . \\
295 . \\
296 . \\
297 . \\
295 . \\
294 .\end{array}$ & $\begin{array}{l}8.310 \\
6.914 \\
3.457 \\
3.145 \\
4.545 \\
6.495 \\
6.636 \\
3.830 \\
5.451 \\
6.638 \\
5.173 \\
2.532 \\
1.349 \\
4.369 \\
4.839 \\
4.152 \\
3.332\end{array}$ & $\begin{array}{l}97.28 \\
23.12 \\
13.06 \\
11.89 \\
12.31 \\
19.92 \\
18.91 \\
15.29 \\
15.31 \\
13.78 \\
14 . \pm 5 \\
: 0.13 \\
7.48 \\
12.28 \\
17.24 \\
19.00 \\
11.60\end{array}$ & $\begin{array}{l}535 . \\
511 . \\
1230 . \\
2327 . \\
328 . \\
1145 . \\
1734 . \\
1314 . \\
13140 \\
1935 . \\
9250 \\
239 . \\
13040 \\
1101 . \\
1645 . \\
1346 . \\
2244 .\end{array}$ & $\begin{array}{r}.600 \\
.600 \\
.600 \\
.600 \\
.600 \\
.600 \\
.571 \\
.600 \\
.600 \\
2.071 \\
.600 \\
.571 \\
.571 \\
.571 \\
.571 \\
.571 \\
.571\end{array}$ & $\begin{array}{r}2=10 . \\
2312 . \\
=30 . \\
1044 . \\
2208 . \\
2400 . \\
2306 . \\
2313 . \\
2241 . \\
\vdots 34 . \\
1950 . \\
1209 . \\
2114 . \\
=20 . \\
2308 . \\
513 . \\
1827 .\end{array}$ \\
\hline 4 & METER & $\begin{array}{l}293 . \\
284 . \\
285 . \\
286 . \\
287 . \\
288 . \\
289 . \\
990 . \\
291 . \\
292 . \\
293 . \\
294 . \\
295 . \\
296 . \\
997 . \\
298 . \\
294 .\end{array}$ & $\begin{array}{l}9.810 \\
8.120 \\
6.260 \\
5.841 \\
5.146 \\
7.351 \\
7.754 \\
4.253 \\
5.965 \\
7.637 \\
5.906 \\
3.197 \\
1.168 \\
1.850 \\
3.499 \\
4.417 \\
3.652\end{array}$ & $\begin{array}{l}27.99 \\
23.15 \\
17.33 \\
13.29 \\
14.18 \\
21.23 \\
21.09 \\
18.76 \\
15.08 \\
16.05 \\
17.91 \\
11.15 \\
7.25 \\
13.47 \\
17.43 \\
21.29 \\
13.04\end{array}$ & 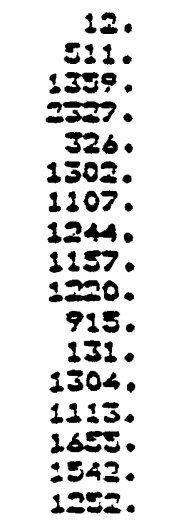 & $\begin{array}{r}.600 \\
.600 \\
.600 \\
.600 \\
.571 \\
.600 \\
. .503 \\
.600 \\
.600 \\
1.873 \\
.600 \\
.571 \\
.571 \\
.571 \\
.571 \\
.571 \\
.571\end{array}$ & $\begin{array}{r}\text { z5s. } \\
2517 . \\
646 . \\
1044 . \\
1954 . \\
2400 . \\
100 . \\
2345 . \\
2241 . \\
134 . \\
2149 . \\
1209 . \\
2114 . \\
222 . \\
2313 . \\
2358 . \\
1827 .\end{array}$ \\
\hline
\end{tabular}


Table 4. Land station: (1) Daily average dry- and wet-bulb air temperatures; and (2) daily maximum and minimum dry-bulb air temperatures and the time they occurred.

[c, degrees celsius]

\begin{tabular}{|c|c|c|c|c|c|c|}
\hline DATE & $\begin{array}{l}\text { DRY BULE } \\
\text { AIR (C) }\end{array}$ & $\begin{array}{l}\text { MAXIMUH } \\
\text { AIR (C) }\end{array}$ & $\begin{array}{l}\text { TIME OF } \\
\text { MAXIMUM }\end{array}$ & $\begin{array}{l}\text { MINIMHA } \\
\text { AIR (e) }\end{array}$ & $\begin{array}{l}\text { TIME OF } \\
\text { KIKIMUH }\end{array}$ & $\begin{array}{l}\text { WET BLLB } \\
\text { AIR (C) }\end{array}$ \\
\hline $\begin{array}{l}150 . \\
151 . \\
152 . \\
1530 \\
2540 \\
i 55 . \\
156 . \\
257 . \\
158 . \\
159 . \\
260 . \\
2610 \\
162 . \\
1630 \\
164 . \\
165 . \\
166 . \\
167 .\end{array}$ & $\begin{array}{l}11.05 \\
12.95 \\
12.80 \\
15.33 \\
14.84 \\
11.71 \\
10.52 \\
13.44 \\
13.50 \\
25.45 \\
14.81 \\
18.73 \\
22.06 \\
20.52 \\
20.53 \\
13.85 \\
10.99 \\
11.76\end{array}$ & $\begin{array}{l}10.56 \\
20.87 \\
21.49 \\
23.86 \\
21.40 \\
16.56 \\
17.18 \\
21.49 \\
24.56 \\
20.45 \\
25.80 \\
29.93 \\
28.52 \\
23.95 \\
25.27 \\
18.23 \\
14.80 \\
19.37\end{array}$ & $\begin{array}{l}1328 . \\
1805 . \\
15 i 3 . \\
1207 . \\
1355 . \\
1619 . \\
3508 . \\
1547 . \\
1513 . \\
1413 . \\
1447 . \\
2450 . \\
1546 . \\
851 . \\
1516 . \\
1859 . \\
929 . \\
1501 .\end{array}$ & $\begin{array}{l}6.98 \\
4.31 \\
6.49 \\
9.26 \\
6.30 \\
3.19 \\
3.57 \\
.56 \\
5.92 \\
2.58 \\
11.90 \\
16.21 \\
17.79 \\
14.89 \\
10.40 \\
9.09 \\
4.95\end{array}$ & $\begin{array}{r}412 . \\
437 . \\
1307 . \\
409 . \\
215 i . \\
443 . \\
22330 \\
0 . \\
438 . \\
2358 . \\
426 . \\
19 . \\
440 \\
120 . \\
2312 . \\
2235 . \\
2359 . \\
2400 .\end{array}$ & $\begin{array}{r}5.50 \\
8.90 \\
6.77 \\
11.42 \\
11.69 \\
9.58 \\
6.92 \\
7.44 \\
7.99 \\
11.47 \\
10.52 \\
15.56 \\
18.20 \\
18.73 \\
18.04 \\
13.03 \\
19.75 \\
9.17\end{array}$ \\
\hline
\end{tabular}

\begin{tabular}{|c|c|c|c|c|c|c|}
\hline DATE & $\begin{array}{c}\text { DSY BULS } \\
\text { AIR }\end{array}$ & $\underset{A I R}{\operatorname{MAXIMUM}}$ & $\begin{array}{l}\text { TIME OF } \\
\text { MAXIMUM }\end{array}$ & MINIMUN & $\begin{array}{l}\text { TIME OF } \\
\text { MINIMUH }\end{array}$ & $\begin{array}{c}\text { UET RULB } \\
\text { AIK }\end{array}$ \\
\hline $\begin{array}{l}168 . \\
169 . \\
270 . \\
171 . \\
172 . \\
273 . \\
174 . \\
175 . \\
176 . \\
177 . \\
176 . \\
179 . \\
180 . \\
181 . \\
182 . \\
183 . \\
184 . \\
185 . \\
186 . \\
187 .\end{array}$ & $\begin{array}{l}15.87 \\
10.02 \\
19.65 \\
20.44 \\
21.45 \\
22.56 \\
20.77 \\
21.72 \\
25.36 \\
21.79 \\
16.12 \\
15.49 \\
14.76 \\
19.44 \\
18.76 \\
18.74 \\
18.50 \\
14.48 \\
15.27 \\
20.55\end{array}$ & $\begin{array}{l}23.42 \\
24.04 \\
26.24 \\
26.59 \\
26.94 \\
27.12 \\
29.23 \\
30.90 \\
31.16 \\
28.43 \\
20.87 \\
24.12 \\
17.44 \\
24.30 \\
26.32 \\
25.39 \\
23.16 \\
30.17 \\
21.05 \\
29.05\end{array}$ & $\begin{array}{l}1524 . \\
1279 . \\
1359 . \\
131: . \\
1709 . \\
1102 . \\
1459 . \\
1508 . \\
1705 . \\
1417 . \\
1613 . \\
1534 . \\
2357 . \\
1425 . \\
1907 . \\
1341 . \\
1110 . \\
1336 . \\
1918 . \\
1426 .\end{array}$ & $\begin{array}{r}2.93 \\
5.57 \\
12.60 \\
16.91 \\
17.62 \\
14.19 \\
10.73 \\
16.03 \\
19.02 \\
13.04 \\
10.05 \\
4.95 \\
13.04 \\
13.83 \\
12.60 \\
14.54 \\
14.71 \\
7.50 \\
4.93 \\
1.47\end{array}$ & $\begin{array}{r}426 . \\
442 . \\
428 . \\
300 . \\
917 . \\
2339 . \\
436 . \\
3 . \\
349 . \\
2356 . \\
2127 . \\
452 . \\
16 . \\
2356 . \\
434 . \\
402 . \\
2352 . \\
2400 . \\
426 . \\
223 .\end{array}$ & $\begin{array}{l}9.55 \\
: 2.83 \\
16.23 \\
19.13 \\
19.53 \\
19.07 \\
16.13 \\
19.53 \\
21.29 \\
18.38 \\
12.38 \\
12.37 \\
13.35 \\
17.29 \\
15.90 \\
17.37 \\
17.95 \\
12.53 \\
9.87 \\
26.96\end{array}$ \\
\hline
\end{tabular}

\begin{tabular}{|c|c|c|c|c|c|c|}
\hline DATE & $\begin{array}{l}\text { DRY BULS } \\
\text { AIR }\end{array}$ & $\begin{array}{c}\text { HAXIMUM } \\
\text { AIR }\end{array}$ & $\begin{array}{l}\text { PIME OF } \\
\text { MAXIMUM }\end{array}$ & $\begin{array}{c}\text { MINIMUM } \\
\text { AIR }\end{array}$ & $\begin{array}{l}\text { TIHE OF } \\
\text { MINIMUM }\end{array}$ & $\begin{array}{l}\text { WET BULS } \\
\text { AIR }\end{array}$ \\
\hline $\begin{array}{l}188 . \\
199 . \\
190 . \\
19: . \\
192 . \\
193 . \\
194 . \\
195 . \\
196 . \\
197 . \\
198 . \\
199 . \\
200 .\end{array}$ & $\begin{array}{l}24.58 \\
26.35 \\
23.83 \\
26.18 \\
29.64 \\
=.30 \\
26.22 \\
20.76 \\
25.87 \\
20.12 \\
19.12 \\
21.38 \\
=.07\end{array}$ & $\begin{array}{l}31.34 \\
38.27 \\
31.16 \\
33.36 \\
33.98 \\
30.99 \\
33.98 \\
33.74 \\
31.16 \\
27.47 \\
35.97 \\
29.05 \\
30.46\end{array}$ & $\begin{array}{l}1636 . \\
1437 . \\
1443 . \\
1458 . \\
\vdots 20 . \\
1651 . \\
1423 . \\
: 349 . \\
1527 . \\
1402 . \\
1830 . \\
: 746 . \\
: 835 .\end{array}$ & $\begin{array}{l}19.02 \\
18.94 \\
19.12 \\
17.88 \\
14.01 \\
12.72 \\
17.18 \\
21.23 \\
20.01 \\
15.77 \\
12.95 \\
14.98 \\
14.62\end{array}$ & $\begin{array}{r}209 . \\
445 . \\
458 . \\
415 . \\
2400 . \\
407 . \\
420 . \\
454 . \\
415 . \\
2352 . \\
457 . \\
1 . \\
502 .\end{array}$ & $\begin{array}{l}20.24 \\
21.93 \\
21.20 \\
21.66 \\
3 . .26 \\
16.60 \\
21.36 \\
20.99 \\
21.99 \\
19.89 \\
17.70 \\
19.40 \\
20.07\end{array}$ \\
\hline
\end{tabular}


Table 4. Land station: (1) Daily average dry- and wet-bulb air temperatures; and (2) daily maximum and minimum dry-bulb air temperatures and the time they occurred. [continued]

\begin{tabular}{|c|c|c|c|c|c|c|}
\hline DATE & $\begin{array}{l}\text { DRY BUE } \\
\text { AIR }\end{array}$ & $\underset{A I R}{\operatorname{MAXIMUM}}$ & $\begin{array}{l}\text { TIME of } \\
\text { MAX:MUY }\end{array}$ & $\begin{array}{c}\text { MENIKUM } \\
\text { AIR }\end{array}$ & $\begin{array}{l}\text { TIME OF } \\
\text { MINIMUH }\end{array}$ & WET RULS \\
\hline $\begin{array}{l}201 . \\
202 . \\
203 . \\
204 . \\
205 . \\
206 . \\
207 . \\
208 . \\
209 . \\
210 . \\
212 . \\
212 . \\
213 . \\
214 . \\
225 . \\
216 .\end{array}$ & $\begin{array}{l}20.21 \\
24.71 \\
25.25 \\
19.20 \\
19.29 \\
29.82 \\
21.75 \\
23.38 \\
23.21 \\
22.83 \\
20.23 \\
28.02 \\
19.76 \\
29.91 \\
24.50 \\
23.92\end{array}$ & $\begin{array}{l}29.31 \\
30.32 \\
29.93 \\
26.39 \\
27.91 \\
28.52 \\
29.40 \\
29.75 \\
30.81 \\
31.51 \\
27.38 \\
24.12 \\
28.87 \\
25.53 \\
32.31 \\
31.51\end{array}$ & $\begin{array}{l}1346 . \\
1418 . \\
1235 . \\
1606 . \\
1821 . \\
18130 \\
1447 . \\
1606 . \\
2500 . \\
1536 . \\
2340 . \\
1636 . \\
1357 . \\
1623 . \\
1439 . \\
1608 .\end{array}$ & $\begin{array}{l}14.98 \\
21.13 \\
10.30 \\
12.25 \\
12.46 \\
10.84 \\
11.81 \\
19.11 \\
17.44 \\
14.10 \\
15.77 \\
12.07 \\
10.40 \\
14.10 \\
18.41 \\
16.65\end{array}$ & $\begin{array}{r}435 . \\
2350 . \\
2400 . \\
459 . \\
454 . \\
424 . \\
513 . \\
235 . \\
2400 . \\
454 . \\
2400 . \\
412 . \\
512 . \\
124 . \\
213 . \\
438 .\end{array}$ & $\begin{array}{l}20.61 \\
22.59 \\
19.61 \\
16.29 \\
16.10 \\
16.14 \\
18.14 \\
20.59 \\
20.56 \\
18.51 \\
18.78 \\
15.43 \\
16.36 \\
18.71 \\
21.77 \\
20.84\end{array}$ \\
\hline
\end{tabular}

\begin{tabular}{|c|c|c|c|c|c|c|}
\hline DATE & $\begin{array}{c}\text { DRY SULS } \\
\text { AIR }\end{array}$ & $\begin{array}{c}\text { MAXIMUH } \\
\text { AIR }\end{array}$ & $\begin{array}{l}\text { TZME OF } \\
\text { MAXIMUM }\end{array}$ & $\begin{array}{c}\text { MINIMUM } \\
\text { AIR }\end{array}$ & $\begin{array}{l}\text { TIKE OF } \\
\text { MINIMUH }\end{array}$ & $\begin{array}{l}\text { WET BULE } \\
\text { AIR }\end{array}$ \\
\hline $\begin{array}{l}217 . \\
218 . \\
219 . \\
220 . \\
221 . \\
222 . \\
223 . \\
224 . \\
228 . \\
226 . \\
227 . \\
228 . \\
229 . \\
130 .\end{array}$ & $\begin{array}{l}25.08 \\
24.51 \\
27.41 \\
21.97 \\
16.88 \\
19.18 \\
18.02 \\
19.90 \\
21.82 \\
20.93 \\
23.68 \\
18.19 \\
23.02 \\
23.51\end{array}$ & $\begin{array}{l}32.48 \\
34.06 \\
35.38 \\
28.43 \\
22.37 \\
24.92 \\
23.36 \\
28.43 \\
27.73 \\
31.07 \\
33.62 \\
23.60 \\
32.49 \\
3: .87\end{array}$ & $\begin{array}{l}1340^{\circ} \\
1723 . \\
1521 . \\
1444 . \\
1439 . \\
1728 . \\
1428 . \\
1404 . \\
1707 . \\
1704 . \\
1803 . \\
1651 . \\
1727 . \\
1509 .\end{array}$ & $\begin{array}{l}17.44 \\
15.13 \\
13.85 \\
19.16 \\
11.11 \\
13.39 \\
10.93 \\
10.05 \\
14.89 \\
10.93 \\
16.12 \\
12.34 \\
17.88 \\
17.79\end{array}$ & $\begin{array}{r}318 . \\
312 . \\
413 . \\
2312 . \\
427 . \\
2253 . \\
2400 . \\
517 . \\
2400 . \\
536 . \\
517 . \\
547 . \\
106 . \\
291 .\end{array}$ & $\begin{array}{l}21.96 \\
29.49 \\
20.40 \\
16.35 \\
15.75 \\
17.02 \\
15.25 \\
26.82 \\
19.49 \\
15.49 \\
21.42 \\
16.94 \\
21.12 \\
21.02\end{array}$ \\
\hline
\end{tabular}

\begin{tabular}{|c|c|c|c|c|c|c|}
\hline DATE & $\begin{array}{c}\text { DRY ZULB } \\
\text { AIR }\end{array}$ & $\begin{array}{c}\text { MAXIMUM } \\
\text { AIR }\end{array}$ & $\begin{array}{l}\text { TIME OF } \\
\text { MAXIMUH }\end{array}$ & $\begin{array}{l}\text { MINIMUM } \\
\text { AIF: }\end{array}$ & $\begin{array}{l}\text { TIME OF } \\
\text { MINIMUA }\end{array}$ & $\begin{array}{c}\text { WET BUL } \\
\text { AIK }\end{array}$ \\
\hline $\begin{array}{l}231 . \\
232 . \\
233 . \\
234 . \\
235 . \\
230 . \\
237 . \\
238 . \\
239 . \\
240 . \\
241 . \\
242 . \\
=43 . \\
244 .\end{array}$ & $\begin{array}{l}19.61 \\
16.79 \\
29.30 \\
17.71 \\
20.17 \\
22.25 \\
25.94 \\
23.40 \\
22.01 \\
24.47 \\
19.19 \\
20.49 \\
20.51 \\
29.00\end{array}$ & $\begin{array}{l}25.97 \\
25.00 \\
26.68 \\
26.68 \\
27.29 \\
29.96 \\
35.30 \\
31.60 \\
33.62 \\
33.62 \\
25.57 \\
30.37 \\
29.84 \\
23.45\end{array}$ & $\begin{array}{l}1427 . \\
1526 . \\
1153 . \\
1317 . \\
1458 . \\
1643 . \\
1356 . \\
1611 . \\
1224 . \\
14530 \\
1430 . \\
1545 . \\
1218 . \\
1547 .\end{array}$ & $\begin{array}{r}8.64 \\
8.03 \\
12.69 \\
9.20 \\
12.16 \\
37.97 \\
16.91 \\
16.38 \\
13.48 \\
10.99 \\
10.50 \\
14.45 \\
14.01 \\
15.15\end{array}$ & $\begin{array}{r}2336 . \\
314 . \\
2400 . \\
517 . \\
209 . \\
521 . \\
446 . \\
2352 . \\
549 . \\
501 . \\
=355 . \\
=354 . \\
100 . \\
521 .\end{array}$ & $\begin{array}{l}13.41 \\
15.00 \\
18.14 \\
16.57 \\
18.77 \\
20.47 \\
24.65 \\
29.28 \\
21.58 \\
29.57 \\
21.57 \\
21.65 \\
20.70 \\
24.19\end{array}$ \\
\hline
\end{tabular}


Table 4. Land station: (1) Daily average dry- and wet-bulb air temperatures; and (2) daily maximum and minimum dry-bulb air temperatures and the time they occurred. [continued]

\begin{tabular}{|c|c|c|c|c|c|c|}
\hline DATE & $\underset{\text { AIR }}{\text { DRY BULB }}$ & $\begin{array}{l}\text { MAXIMUA } \\
\text { AIR }\end{array}$ & $\begin{array}{l}\text { TIKE of } \\
\text { MAXIMUY }\end{array}$ & $\begin{array}{l}\text { MINIMUY } \\
\text { AIK }\end{array}$ & $\begin{array}{l}\text { TIME OF } \\
\text { MINIMUM }\end{array}$ & $\begin{array}{c}\text { WET BULE } \\
\text { AIK }\end{array}$ \\
\hline $\begin{array}{l}245 . \\
246 . \\
247 . \\
248 . \\
248 . \\
250 . \\
251 . \\
252 . \\
253 . \\
254 . \\
255 . \\
256 . \\
257 . \\
258 . \\
259 . \\
260 . \\
261 . \\
262 .\end{array}$ & $\begin{array}{r}27.53 \\
21.43 \\
27.23 \\
10.29 \\
12.75 \\
197.96 \\
23.38 \\
19.58 \\
15.07 \\
7.96 \\
10.34 \\
10.09 \\
9.74 \\
10.45 \\
10.47 \\
22.70 \\
9.80 \\
6.99\end{array}$ & $\begin{array}{l}57 .=3 \\
26.50 \\
24.39 \\
25.18 \\
16.39 \\
26.35 \\
36.90 \\
26.24 \\
13.94 \\
11.90 \\
17.18 \\
18.76 \\
17.35 \\
11.11 \\
13.92 \\
17.00 \\
14.98 \\
10.49\end{array}$ & 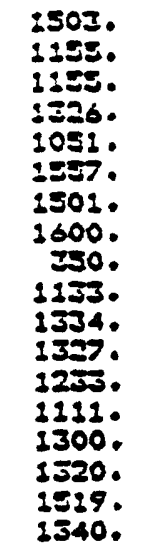 & $\begin{array}{r}17.88 \\
14.61 \\
12.25 \\
10.31 \\
8.39 \\
12.57 \\
10.35 \\
15.42 \\
8.38 \\
3.02 \\
4.07 \\
2.76 \\
1.197 \\
9.26 \\
8.64 \\
9.32 \\
3.55 \\
4.07\end{array}$ & $\begin{array}{r}600 . \\
2400 . \\
235: . \\
54 ! . \\
558 . \\
435 . \\
209 . \\
2517 . \\
2357 . \\
553 . \\
540 . \\
2400 . \\
538 . \\
2400 . \\
439 . \\
8 . \\
2245 . \\
155 .\end{array}$ & $\begin{array}{r}27.57 \\
21.69 \\
17.42 \\
16.61 \\
12.97 \\
13.23 \\
23.59 \\
19.83 \\
15.41 \\
8.13 \\
10.05 \\
10.39 \\
9.94 \\
10.64 \\
20.68 \\
12.94 \\
10.13 \\
7.18\end{array}$ \\
\hline
\end{tabular}

\begin{tabular}{|c|c|c|c|c|c|c|}
\hline DATE & $\begin{array}{c}\text { DRY BULB } \\
\text { AIR }\end{array}$ & $\begin{array}{c}\text { MaXImuM } \\
\text { AIF. }\end{array}$ & $\begin{array}{l}\text { TIME OF } \\
\text { MAXIMUM }\end{array}$ & $\begin{array}{c}\text { MINIKUM } \\
\text { AIRี }\end{array}$ & $\begin{array}{l}\text { TIME OF } \\
\text { MINIMUH }\end{array}$ & $\begin{array}{c}\text { WET BULS } \\
\text { AIR }\end{array}$ \\
\hline $\begin{array}{l}265 . \\
264 . \\
265 . \\
260 . \\
267 . \\
268 . \\
269 . \\
270 . \\
271 . \\
272 . \\
275 . \\
274 . \\
275 . \\
276 . \\
277 . \\
278 . \\
270 . \\
290 . \\
281 . \\
252 .\end{array}$ & $\begin{array}{r}3.44 \\
4.70 \\
2.88 \\
8.00 \\
11.41 \\
14.38 \\
13.79 \\
16.93 \\
18.90 \\
10.11 \\
15.04 \\
14.30 \\
9.88 \\
8.98 \\
9.44 \\
8.06 \\
6.07 \\
3.43 \\
4.08 \\
7.50\end{array}$ & $\begin{array}{r}7.85 \\
8.82 \\
6.77 \\
10.30 \\
21.05 \\
36.32 \\
26.32 \\
27.03 \\
23.55 \\
12.50 \\
19.02 \\
18.76 \\
12.34 \\
10.67 \\
14.01 \\
11.75 \\
13.83 \\
7.94 \\
16.03 \\
14.54\end{array}$ & $\begin{array}{r}1310 . \\
13430 \\
1219 . \\
1544 . \\
1555 . \\
1601 . \\
1455 . \\
1=57 . \\
1232 . \\
6 . . \\
2217 . \\
29 . \\
1214 . \\
1427 . \\
1251 . \\
1055 . \\
1550 . \\
408 . \\
1617 . \\
1420 .\end{array}$ & $\begin{array}{r}4.34 \\
2.76 \\
-3.05 \\
-3.31 \\
3.46 \\
1.35 \\
4.16 \\
4.95 \\
22.69 \\
8.38 \\
20.67 \\
9.35 \\
8.82 \\
6.98 \\
7.41 \\
2.76 \\
-1.81 \\
-1.35 \\
-3.13 \\
-2.34\end{array}$ & $\begin{array}{r}445 . \\
2453 . \\
2452 . \\
556 . \\
2545 . \\
624 . \cdot \\
631 . \\
335 . \\
2400 . \\
712 . \\
29 . \\
2536 . \\
630 . \\
2327 . \\
2=23 . \\
2400 . \\
624 . \\
2400 . \\
437 . \\
103 .\end{array}$ & $\begin{array}{r}5.67 \\
4.94 \\
5.06 \\
6.14 \\
11.74 \\
14.29\end{array}$ \\
\hline
\end{tabular}

\begin{tabular}{|c|c|c|c|c|c|c|}
\hline DATE & $\begin{array}{l}\text { DRY BULA } \\
\text { AIR }\end{array}$ & $\begin{array}{l}\text { MAXIMUM } \\
\text { AIK }\end{array}$ & $\begin{array}{l}\text { TIME OF } \\
\text { MAXIHUM }\end{array}$ & $\begin{array}{l}\text { MINIMUM } \\
\text { AIR }\end{array}$ & $\begin{array}{l}\text { TIME OF } \\
\text { MINJMUH }\end{array}$ & $\begin{array}{c}\text { HET BULB } \\
\text { AIR }\end{array}$ \\
\hline $\begin{array}{l}283 . \\
294 . \\
285 . \\
296 . \\
295 . \\
298 . \\
298 . \\
239 . \\
290 . \\
291 . \\
292 . \\
293 . \\
294 . \\
295 . \\
296 . \\
297 . \\
298 . \\
299 .\end{array}$ & $\begin{array}{l}9.29 \\
5.71 \\
1.43 \\
2.05 \\
4.32 . \\
4.95 \\
4.62 \\
4.60 \\
3.85 \\
5.93 \\
5.53 \\
6.36 \\
7.25 \\
3.00 \\
5.08 \\
4.59 \\
6.05\end{array}$ & $\begin{array}{r}12.37 \\
11.72 \\
7.30 \\
3.55 \\
14.10 \\
5.85 \\
9.79 \\
12.10 \\
5.29 \\
7.50 \\
12.60 \\
10.49 \\
16.91 \\
9.09 \\
10.31 \\
14.36 \\
15.42\end{array}$ & $\begin{array}{r}2400 . \\
138 . \\
1422 . \\
1257 . \\
1541 . \\
2400 . \\
1457 . \\
1348 . \\
5 . \\
1241 . \\
1434 . \\
1327 . \\
2500 . \\
15410 \\
1530 . \\
24390 \\
1519 .\end{array}$ & $\begin{array}{r}7.68 \\
-3.84 \\
-1.46 \\
.38 \\
-1.64 \\
3.72 \\
-1.73 \\
-2.45 \\
2.84 \\
3.90 \\
.47 \\
3.63 \\
-.23 \\
-1.99 \\
-1.95 \\
-4.19 \\
-2.78\end{array}$ & $\begin{array}{r}1111 . \\
2257 . \\
530 . \\
611 . \\
2137 . \\
7040 \\
2=58 . \\
5420 \\
7200 \\
40 \\
20550 \\
25150 \\
4040 \\
2400 . \\
630 . \\
515 .\end{array}$ & . \\
\hline
\end{tabular}


Table 5. Land station: (1) Daily average wind speed;

(2) daily maximum and minimum wind speed and the time they occurred; and (3) daily total precipitation.

[mph, miles per hour; in, inches]

\begin{tabular}{|c|c|c|c|c|c|c|}
\hline DATE & $\begin{array}{l}\text { WINDEQA } \\
\text { IIAILY } \\
\text { AUE (mpN) }\end{array}$ & $\begin{array}{l}\text { UIXDean } \\
\text { DAI:Y } \\
\text { InX (inph) }\end{array}$ & 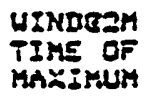 & $\begin{array}{l}\text { WENDEZM } \\
\text { DAILY } \\
\text { MIN: PAph }\end{array}$ & $\begin{array}{l}\text { WTNDE2H } \\
\text { TIRE OF } \\
\text { MINIXUH }\end{array}$ & $\begin{array}{l}\text { PREEIP } \\
\text { TCTAL (In) }\end{array}$ \\
\hline $\begin{array}{l}: 30 . \\
15: . \\
152 . \\
\vdots 530 \\
254 . \\
155 . \\
156 . \\
157 . \\
158 . \\
159 . \\
160 . \\
161 . \\
162 . \\
163 . \\
104 . \\
165 . \\
266 . \\
167 .\end{array}$ & $\begin{array}{l}3.364 \\
2.362 \\
1.982 \\
1.794 \\
3.398 \\
3.697 \\
3.077 \\
3.570 \\
2.602 \\
3.823 \\
2.190 \\
3.117 \\
2.641 \\
2.842 \\
3.770 \\
2.797 \\
6.409 \\
3.914\end{array}$ & $\begin{array}{r}19.67 \\
15.08 \\
12.20 \\
9.76 \\
20.63 \\
13.87 \\
36.08 \\
27.20 \\
12.08 \\
17.46 \\
9.99 \\
12.96 \\
10.39 \\
11.07 \\
16.10 \\
14.04 \\
20.60 \\
13.30\end{array}$ & $\begin{array}{l}1250 . \\
1151 . \\
1453 . \\
1917 . \\
1329 . \\
303 . \\
1046 . \\
1647 . \\
1720 . \\
1209 . \\
\$ 732 . \\
1201 . \\
1617 . \\
555 . \\
234 . \\
1024 . \\
1403 . \\
1041 .\end{array}$ & $\begin{array}{l}.350 \\
.343 \\
.543 \\
.600 \\
.600 \\
.600 \\
.571 \\
.571 \\
.571 \\
.571 \\
.571 \\
.600 \\
.600 \\
.600 \\
.600 \\
.571 \\
.571 \\
.571\end{array}$ & $\begin{array}{r}1602 . \\
955 . \\
1651 . \\
2400 . \\
2325 . \\
2400 . \\
548 . \\
2250 . \\
343 . \\
2259 . \\
545 . \\
2239 . \\
2400 . \\
2130 . \\
2358 . \\
2225 . \\
129 . \\
2106 .\end{array}$ & $\begin{array}{l}0.00 \\
0.00 \\
0.00 \\
0.00 \\
.09 \\
.002 \\
0.00 \\
0.00 \\
0.00 \\
.01 \\
0.00 \\
.07 \\
.07 \\
.83 \\
2.00 \\
1.44 \\
.01 \\
0.00\end{array}$ \\
\hline
\end{tabular}

\begin{tabular}{|c|c|c|c|c|c|c|}
\hline DATE & $\begin{array}{l}\text { WINDRZM } \\
\text { DAILY } \\
\text { AUERAGE }\end{array}$ & $\begin{array}{l}\text { WINDEZM } \\
\text { DAIIY } \\
\text { MUXIMUM }\end{array}$ & $\begin{array}{l}\text { WINDEZM } \\
\text { TIME OF } \\
\text { MAXIMUH }\end{array}$ & $\begin{array}{l}\text { WENDEבM } \\
\text { DAILY } \\
\text { MINIMUM }\end{array}$ & $\begin{array}{l}\text { HINDE2M } \\
\text { TIME OF } \\
\text { MINIMUM }\end{array}$ & $\begin{array}{l}\text { PEEEIF. } \\
\text { TOTAL }\end{array}$ \\
\hline $\begin{array}{l}168 . \\
169 . \\
170 . \\
171 . \\
172 . \\
173 . \\
174 . \\
175 . \\
176 . \\
177 \circ \\
1780 \\
179 . \\
180 . \\
181 . \\
182 . \\
183 . \\
184 . \\
185 . \\
186 . \\
187 .\end{array}$ & $\begin{array}{l}2.823 \\
2.888 \\
3.404 \\
2.924 \\
2.490 \\
5.442 \\
2.058 \\
2.100 \\
3.853 \\
1.765 \\
5.151 \\
3.697 \\
4.029 \\
4.139 \\
2.806 \\
3.247 \\
2.852 \\
5.826 \\
2.290 \\
2.366\end{array}$ & $\begin{array}{r}7.98 \\
19.29 \\
13.99 \\
11.04 \\
10.59 \\
21.45 \\
8.77 \\
10.27 \\
17.41 \\
12.93 \\
14.29 \\
13.90 \\
11.29 \\
15.92 \\
12.65 \\
14.38 \\
20.86 \\
18.45 \\
13.27 \\
15.20\end{array}$ & $\begin{array}{l}1334 . \\
1424 . \\
1423 . \\
1=34 . \\
3353 . \\
1439 . \\
1442 . \\
541 . \\
1158 . \\
1349 . \\
=39 . \\
1416 . \\
2918 \\
1652 . \\
1314 . \\
2040 . \\
=955 . \\
727 . \\
1434 . \\
1546 .\end{array}$ & $\begin{array}{l}.571 \\
.600 \\
.600 \\
.600 \\
.600 \\
.571 \\
.571 \\
.572 \\
.600 \\
.571 \\
.543 \\
.600 \\
.600 \\
.600 \\
.600 \\
.600 \\
.600 \\
.600 \\
.571 \\
.515\end{array}$ & $\begin{array}{r}404 . \\
2342 . \\
2334 . \\
2359 . \\
2352 . \\
2340 . \\
516 . \\
317 . \\
2357 . \\
343 . \\
27 . \\
2226 . \\
2098 . \\
2359 . \\
2359 . \\
2336 . \\
2047 . \\
2400 . \\
644 . \\
944 .\end{array}$ & $\begin{array}{l}0.00 \\
0.00 \\
0.00 \\
0.00 \\
.35 \\
0.00 \\
0.00 \\
.021 \\
0.00 \\
.26 \\
0.00 \\
0.00 \\
.76 \\
.42 \\
0.00 \\
3.56 \\
.12 \\
.15 \\
0.00 \\
0.00\end{array}$ \\
\hline
\end{tabular}

\begin{tabular}{|c|c|c|c|c|c|c|}
\hline DATE & $\begin{array}{l}\text { WHREZM } \\
\text { DAILY } \\
\text { AUEARGE }\end{array}$ & $\begin{array}{l}\text { WINDEAM } \\
\text { MAILY } \\
\text { MAXIMUM }\end{array}$ & $\begin{array}{l}\text { WINDEZH } \\
\text { TIME OF } \\
\text { MAXIMUH }\end{array}$ & $\begin{array}{l}\text { UINDEZM } \\
\text { DAILY } \\
\text { MINIMUM }\end{array}$ & $\begin{array}{l}\text { HIMDEZH } \\
\text { TIME of } \\
\text { MIMIMUH }\end{array}$ & $\begin{array}{l}\text { PREEIP. } \\
\text { TOTAL }\end{array}$ \\
\hline $\begin{array}{l}188 . \\
189 . \\
: 90 . \\
: 91 . \\
: 92 . \\
195 . \\
194 . \\
195 . \\
196 . \\
197 . \\
198 . \\
199 . \\
200 .\end{array}$ & $\begin{array}{l}2.985 \\
2.464 \\
3.932 \\
2.807 \\
6.629 \\
2.816 \\
2.525 \\
2.213 \\
2.401 \\
2.701 \\
2.018 \\
1.974 \\
1.768\end{array}$ & $\begin{array}{r}13.73 \\
9.29 \\
10.08 \\
13.95 \\
25.57 \\
13.76 \\
10.42 \\
11.46 \\
9.76 \\
11.58 \\
11.01 \\
9.14 \\
10.08\end{array}$ & $\begin{array}{l}402 . \\
2342 . \\
2040 . \\
1309 . \\
1424 . \\
1247 . \\
1438 . \\
1=24 . \\
1705 . \\
59 . \\
1330 . \\
1148 . \\
1035 .\end{array}$ & $\begin{array}{l}.600 \\
.572 \\
.600 \\
.571 \\
.571 \\
.600 \\
.600 \\
.625 \\
.600 \\
.600 \\
.600 \\
.600 \\
.571\end{array}$ & $\begin{array}{r}9311 . \\
345 . \\
1914 . \\
2104 . \\
2046 . \\
2400 . \\
2359 . \\
3231 . \\
2341 . \\
2400 . \\
2400 . \\
2400 . \\
253 .\end{array}$ & $\begin{array}{l}0.00 \\
0.00 \\
0.00 \\
0.00 \\
0.00 \\
0.00 \\
0.00 \\
0.00 \\
0.00 \\
.47 \\
.38 \\
.01 \\
.02\end{array}$ \\
\hline
\end{tabular}


Table 5. Land station: (1) Daily average wind speed; (2) daily maximum and minimum wind speed and the time they occurred; and (3) daily total precipitation. [continued]

\begin{tabular}{|c|c|c|c|c|c|c|}
\hline DATE & $\begin{array}{l}\text { WINDEEY } \\
\text { IAILY } \\
\text { AUERAGE }\end{array}$ & $\begin{array}{l}\text { HINDEבH } \\
\text { JAI:LY } \\
\text { MAXIMUH }\end{array}$ & $\begin{array}{l}\text { LINDEZM } \\
\text { TISE OF } \\
\text { MAXIMUK }\end{array}$ & $\begin{array}{l}\text { WINDEZM } \\
\text { MAILY } \\
\text { HINIKUM }\end{array}$ & $\begin{array}{l}\text { WINDEIM } \\
\text { TIME OF } \\
\text { MINIXUH }\end{array}$ & $\begin{array}{l}\text { PREEIF. } \\
\text { TOTAL }\end{array}$ \\
\hline $\begin{array}{l}201 . \\
202 . \\
205 . \\
204 . \\
205 . \\
206 . \\
207 . \\
208 . \\
209 . \\
210 . \\
211 . \\
212 . \\
213 . \\
214 . \\
215 . \\
216 .\end{array}$ & $\begin{array}{l}2.396 \\
3.247 \\
2.833 \\
3.051 \\
1.735 \\
1.850 \\
2.084 \\
1.923 \\
1.925 \\
2.107 \\
2.045 \\
3.759 \\
1.705 \\
2.000 \\
2.244 \\
1.589\end{array}$ & $\begin{array}{r}20.29 \\
22.85 \\
34.46 \\
18.54 \\
10.25 \\
6.32 \\
11.58 \\
9.45 \\
10.92 \\
9.31 \\
22.00 \\
15.54 \\
7.25 \\
9.40 \\
11.43 \\
6.20\end{array}$ & $\begin{array}{l}940 . \\
1119 . \\
1575 . \\
956 . \\
1146 . \\
1040 . \\
1035 . \\
554 . \\
1715 . \\
1250 . \\
549 . \\
1251 . \\
1043 . \\
1540 . \\
1306 . \\
1116 .\end{array}$ & $\begin{array}{l}.545 \\
.600 \\
.571 \\
.000 \\
.600 \\
.571 \\
.458 \\
.571 \\
.571 \\
.571 \\
.571 \\
.600 \\
.345 \\
.571 \\
.034 \\
.543\end{array}$ & $\begin{array}{r}1155 . \\
2358 . \\
2314 . \\
2400 . \\
2400 . \\
125 . \\
1357 . \\
2400 . \\
2347 . \\
603 . \\
154 . \\
2400 . \\
2348 . \\
350 . \\
2400 . \\
2058 .\end{array}$ & $\begin{array}{l}.05 \\
0.00 \\
0.00 \\
0.00 \\
0.00 \\
0.00 \\
0.00 \\
0.00 \\
0.00 \\
0.00 \\
.04 \\
0.00 \\
0.00 \\
.111 \\
0.00 \\
0.00\end{array}$ \\
\hline
\end{tabular}

\begin{tabular}{|c|c|c|c|c|c|c|}
\hline DATE & $\begin{array}{l}\text { WINDEZM } \\
\text { DAILY } \\
\text { AUERAGE }\end{array}$ & $\begin{array}{l}\text { WINDEZM } \\
\text { DAILY } \\
\text { MAXINUM }\end{array}$ & $\begin{array}{l}\text { WINDE2M } \\
\text { TIME OF } \\
\text { MAXINUM }\end{array}$ & $\begin{array}{l}\text { UINDEAM } \\
\text { DAILY } \\
\text { KINIMUM }\end{array}$ & $\begin{array}{l}\text { HINDEAM } \\
\text { TIME OF } \\
\text { MINIMUM }\end{array}$ & $\begin{array}{l}\text { PREEIP. } \\
\text { TOTAL }\end{array}$ \\
\hline $\begin{array}{l}217 . \\
218 . \\
219 . \\
220 . \\
221 . \\
229 . \\
223 . \\
224 . \\
225 . \\
226 . \\
227 . \\
228 . \\
229 . \\
230 .\end{array}$ & $\begin{array}{l}2.442 \\
1.701 \\
4.962 \\
2.373 \\
1.669 \\
3.137 \\
1.420 \\
2.112 \\
2.363 \\
1.034 \\
1.354 \\
2.353 \\
1.421 \\
1.776\end{array}$ & $\begin{array}{r}11.52 \\
7.30 \\
24.03 \\
12.54 \\
9.11 \\
12.82 \\
10.73 \\
9.29 \\
13.78 \\
6.94 \\
8.38 \\
13.11 \\
7.08 \\
9.14\end{array}$ & $\begin{array}{r}1257 . \\
1148 . \\
1349 . \\
1351 . \\
21040 \\
1707 . \\
846 . \\
1333 . \\
1442 . \\
10170 \\
1012 . \\
1432 . \\
14340 \\
21: 7 .\end{array}$ & $\begin{array}{l}.543 \\
.571 \\
.600 \\
.600 \\
.458 \\
.600 \\
.600 \\
.288 \\
.571 \\
.571 \\
.600 \\
.571 \\
.543 \\
.571\end{array}$ & $\begin{array}{r}212 . \\
643 . \\
2400 . \\
2400 . \\
1227 . \\
2400 . \\
2400 . \\
801 . \\
2035 . \\
2346 . \\
2400 . \\
1951 . \\
1210 . \\
2219 .\end{array}$ & $\begin{array}{l}0.00 \\
0.00 \\
0.00 \\
0.00 \\
.20 \\
.00 \\
0.00 \\
0.00 \\
.07 \\
0.00 \\
0.00 \\
.32 \\
0.00 \\
0.00\end{array}$ \\
\hline
\end{tabular}

\begin{tabular}{|c|c|c|c|c|c|c|}
\hline BATE & $\begin{array}{l}\text { WINDEZM } \\
\text { DAILY } \\
\text { AUERASE }\end{array}$ & $\begin{array}{l}\text { WINDEZM } \\
\text { DAILY } \\
\text { MAXIMUM }\end{array}$ & $\begin{array}{l}\text { WINDRZM } \\
\text { TIHE OF } \\
\text { MAXIMUM }\end{array}$ & $\begin{array}{l}\text { WINDEZM } \\
\text { DAILY } \\
\text { MINIMUM }\end{array}$ & $\begin{array}{l}\text { HINDE2H } \\
\text { IIHE OF } \\
\text { MINIMUH }\end{array}$ & $\begin{array}{l}\text { PREETP. } \\
\text { TOTAL }\end{array}$ \\
\hline $\begin{array}{l}231 . \\
232 . \\
233 . \\
234 . \\
235 . \\
236 . \\
237 . \\
238 . \\
239 . \\
240 . \\
241 . \\
242 . \\
243 . \\
244 .\end{array}$ & $\begin{array}{l}3.084 \\
1.989 \\
2.629 \\
1.476 \\
2.074 \\
=.717 \\
3.155 \\
2.014 \\
1.929 \\
3.063 \\
2.542 \\
2.240 \\
1.663 \\
2.352\end{array}$ & $\begin{array}{r}16.47 \\
9.88 \\
12.18 \\
8.92 \\
9.93 \\
: 3.02 \\
10.52 \\
32.38 \\
11.05 \\
12.08 \\
9.65 \\
13.30 \\
10.81 \\
12.59\end{array}$ & $\begin{array}{r}1442 . \\
1654 . \\
1601 . \\
1332 . \\
1326 . \\
941 . \\
247 . \\
: 33 . \\
2353 . \\
1357 . \\
2832 . \\
1254 . \\
1214 . \\
1034 .\end{array}$ & $\begin{array}{l}.543 \\
.571 \\
.600 \\
.571 \\
.600 \\
.600 \\
.600 \\
.571 \\
.600\end{array}$ & $\begin{array}{r}121 . \\
726 . \\
2400 . \\
2302 . \\
2400 . \\
1136 . \\
2302 . \\
2400 . \\
120 . \\
2400 .\end{array}$ & $\begin{array}{l}0.00 \\
0.00 \\
.01 \\
0.00 \\
0.00 \\
0.00 \\
0.00 \\
.10 \\
0.00 \\
0.00 \\
.10 \\
0.00 \\
.1= \\
0.00\end{array}$ \\
\hline
\end{tabular}


Table 5. Land station: (1) Daily average wind speed; (2) daily maximum and minimum wind speed and the time they occurred; and (3) daily total precipitation. [continued]

\begin{tabular}{|c|c|c|c|c|c|c|}
\hline DATE & $\begin{array}{l}\text { WINDEQY } \\
\text { DAILY } \\
\text { AUERACE }\end{array}$ & $\begin{array}{l}\text { WIKERAM } \\
\text { IAIEY } \\
\text { MAXIMUN }\end{array}$ & $\begin{array}{l}\text { HINDEZM } \\
\text { TIKE OF } \\
\text { MAXIKL: }\end{array}$ & $\begin{array}{l}\text { LINDEZM } \\
\text { IAILY } \\
\text { MINIHUM }\end{array}$ & $\begin{array}{l}\text { HINDQZM } \\
\text { PIME OF } \\
\text { HINIMUK }\end{array}$ & $\begin{array}{l}\text { PRESIP. } \\
\text { TOTAL }\end{array}$ \\
\hline $\begin{array}{l}245 . \\
240 . \\
247 . \\
248 . \\
245 . \\
250 . \\
251 . \\
252 . \\
253 . \\
254 . \\
255 . \\
256 . \\
257 . \\
258 . \\
259 . \\
260 . \\
261 . \\
262 .\end{array}$ & $\begin{array}{l}2.59= \\
1.001 \\
1.018 \\
0.052 \\
5.069 \\
2.79: \\
2.399 \\
2.003 \\
6.799 \\
1.390 \\
2.135 \\
1.610 \\
2.224 \\
5.250 \\
3.709 \\
3.353 \\
6.650 \\
2.013\end{array}$ & $\begin{array}{r}19 .=5 \\
6.57 \\
3.35 \\
19.30 \\
23.25 \\
11.55 \\
17.97 \\
10.30 \\
23.05 \\
7.15 \\
8.49 \\
8.80 \\
10.95 \\
14.24 \\
11.18 \\
-2.62 \\
22.27 \\
8.04\end{array}$ & 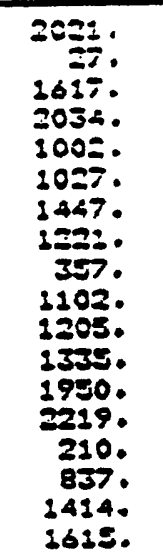 & & & $\begin{array}{l}0.02 \\
0.08 \\
.02 \\
.05 \\
.01 \\
0.00 \\
0.00 \\
0.00 \\
.29 \\
.18 \\
.01 \\
0.00 \\
.03 \\
.95 \\
0.00 \\
.06 \\
0.00 \\
.02\end{array}$ \\
\hline
\end{tabular}

\begin{tabular}{|c|c|c|c|c|c|c|}
\hline DATE & $\begin{array}{l}\text { WINDEZM } \\
\text { DAILY } \\
\text { AUERAEE }\end{array}$ & $\begin{array}{l}\text { WINREZM } \\
\text { JAILY } \\
\text { MAXIMUM }\end{array}$ & $\begin{array}{l}\text { WINDEZM } \\
\text { TIME OF } \\
\text { MAXIHUH }\end{array}$ & $\begin{array}{l}\text { WINDEAM } \\
\text { JAIILY } \\
\text { MINIMUM }\end{array}$ & $\begin{array}{l}\text { WINDEZM } \\
\text { TIHE OF } \\
\text { MINIMUH. }\end{array}$ & $\begin{array}{l}\text { PREEIF. } \\
\text { TOTAL }\end{array}$ \\
\hline $\begin{array}{l}253 . \\
265 . \\
265 . \\
266 . \\
267 . \\
268 . \\
269 . \\
370 . \\
27.0 \\
972 . \\
273 . \\
274 . \\
275 . \\
270 . \\
277 . \\
278 . \\
279 . \\
280 . \\
281 . \\
282 .\end{array}$ & $\begin{array}{l}6.493 \\
6.494 \\
4.589 \\
2.191 \\
2.544 \\
1.738 \\
1.580 \\
2.254 \\
3.297 \\
3.542 \\
1.793 \\
2.269 \\
2.735 \\
2.932 \\
1.478 \\
5.570 \\
2.829 \\
3.699 \\
1.260 \\
2.646\end{array}$ & $\begin{array}{r}17.55 \\
17.15 \\
18.00 \\
15.57 \\
12.11 \\
15.11 \\
8.69 \\
10.56 \\
12.23 \\
10.16 \\
9.20 \\
9.17 \\
14.94 \\
10.47 \\
5.86 \\
17.35 \\
16.19 \\
14.18 \\
8.38 \\
16.95\end{array}$ & 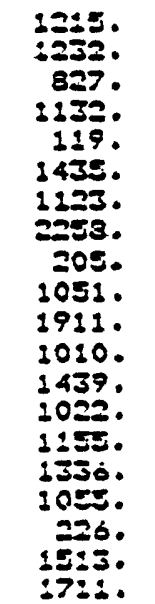 & & & $\begin{array}{l}.03 \\
.03 \\
0.00 \\
0.00 \\
0.00 \\
0.00 \\
0.00 \\
0.00 \\
0.00 \\
.03 \\
.19 \\
.25 \\
.07 \\
.03 \\
.12 \\
0.00 \\
0.00 \\
.04 \\
0.00 \\
0.00\end{array}$ \\
\hline
\end{tabular}

\begin{tabular}{|c|c|c|c|c|c|c|}
\hline DATE & $\begin{array}{l}\text { WINDEZM } \\
\text { DAILY } \\
\text { AUEAAGE }\end{array}$ & $\begin{array}{l}\text { WIND82H } \\
\text { DAILY } \\
\text { MAXIMUH }\end{array}$ & $\begin{array}{l}\text { WINRE } \\
\text { TIME OF } \\
\text { MAXIMUM }\end{array}$ & $\begin{array}{l}\text { WIMDEZM } \\
\text { BAILY } \\
\text { HINIMUM }\end{array}$ & $\begin{array}{l}\text { WINDEZM } \\
\text { TIKE OF } \\
\text { MINIMUH }\end{array}$ & $\begin{array}{l}\text { PRESIP. } \\
\text { TOTAL }\end{array}$ \\
\hline $\begin{array}{l}293 . \\
294 . \\
295 . \\
295 . \\
290 . \\
287 . \\
298 . \\
299 . \\
290 . \\
295 . \\
292 . \\
293 . \\
294 . \\
295 . \\
290 . \\
297 . \\
299 . \\
299 .\end{array}$ & $\begin{array}{l}2.307 \\
5.105 \\
3.035 \\
2.683 \\
3.457 \\
0.552 \\
5.596 \\
2.775 \\
6.685 \\
5.840 \\
3.424 \\
1.738 \\
\vdots .145 \\
1.505 \\
2.715 \\
2.575 \\
2.249\end{array}$ & $\begin{array}{r}16.27 \\
20.55 \\
17.91 \\
9.25 \\
10.61 \\
23.57 \\
21.00 \\
16.70 \\
16.30 \\
12.99 \\
22.85 \\
7.30 \\
6.34 \\
8.38 \\
13.07 \\
19.92 \\
15.27\end{array}$ & $\begin{array}{r}251 . \\
444 . \\
1340 . \\
=3130 \\
20 . \\
=31 . \\
1354 . \\
3: 250 \\
1024 . \\
419 . \\
530 . \\
47 . \\
1347 . \\
1057 . \\
1035 . \\
1326 . \\
1210 .\end{array}$ & & & $\begin{array}{l}.35 \\
0.00 \\
.01 \\
.11 \\
0.00 \\
.13 \\
.10 \\
0.00 \\
0.00 \\
.05 \\
.03 \\
0.00 \\
0.00 \\
0.00 \\
.05 \\
0.00 \\
0.00\end{array}$ \\
\hline
\end{tabular}


Table 6. Land station: (1) Daily total short- and long-wave radiation;

(2) daily maximum short-wave radiation and the time it occurred; and (3) daily maximum and minimum long-wave radiation and the time they occurred.

[cal/ $\mathrm{cm}^{2} /$ day, calories per square centimenter per day]

\begin{tabular}{|c|c|c|c|c|c|c|c|c|}
\hline DATE & $\begin{array}{c}\text { SHORT } \\
\text { WAVE } \\
\text { TOTAL } \\
\text { feal/cm/doy }\end{array}$ & $\begin{array}{c}\text { SHORT } \\
\text { WAUE } \\
\text { MnXimun } \\
\text { (col/ } \mathrm{cm}^{2} / \text { day) }\end{array}$ & $\begin{array}{l}\text { ilne of } \\
\text { SHort } \\
\text { MnXImUn }\end{array}$ & $\begin{array}{l}\text { LONE } \\
\text { WAVE } \\
\text { TOTAL } \\
\left.\text { (col/cm } / \mathrm{cm}^{2} / \mathrm{dO}\right)\end{array}$ & $\begin{array}{l}\text { LONG } \\
\text { WAUE } \\
\text { MnXInius } \\
\text { (cal/cmiday) }\end{array}$ & $\begin{array}{l}\text { TIME OF } \\
\text { LONG } \\
\text { MAXIMUN }\end{array}$ & $\begin{array}{c}\text { LOMG } \\
\text { WNUE } \\
\text { MINIMUK } \\
\text { (col/emdoy) }\end{array}$ & $\begin{array}{l}\text { TIME OF } \\
\text { LONG } \\
\text { MIMIMUN }\end{array}$ \\
\hline $\begin{array}{l}150 . \\
151 . \\
152 . \\
153 . \\
154 . \\
155 . \\
156 . \\
157 . \\
153 . \\
159 . \\
160 . \\
161 . \\
162 . \\
365 . \\
164 . \\
165 . \\
166 . \\
167 .\end{array}$ & $\begin{array}{l}384.7 \\
644.6 \\
431.7 \\
418.5 \\
566.6 \\
293.6 \\
365.1 \\
718.9 \\
714.4 \\
522.8 \\
686.8 \\
459.5 \\
531.7 \\
222.4 \\
280.5 \\
172.1 \\
291.3 \\
522.6\end{array}$ & $\begin{array}{l}1.838 \\
1.703 \\
6.935 \\
1.674 \\
1.654 \\
1.846 \\
1.757 \\
1.370 \\
1.352 \\
1.646 \\
1.355 \\
1.624 \\
1.537 \\
1.279 \\
1.641 \\
.983 \\
1.741 \\
1.839\end{array}$ & 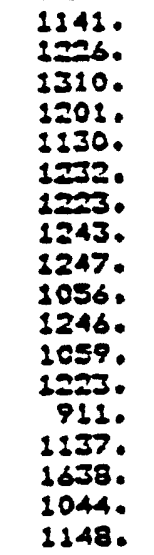 & $\begin{array}{l}634.0 \\
589.8 \\
700.8 \\
638.7 \\
653.8 \\
643.8 \\
573.6 \\
542.4 \\
502.9 \\
575.3 \\
576.8 \\
710.8 \\
753.6 \\
653.2\end{array}$ & $\begin{array}{l}.510 \\
.507 \\
.554 \\
.542 \\
.517 \\
.477 \\
.453 \\
.428 \\
.028 \\
.477 \\
.582 \\
.589 \\
.528\end{array}$ & $\begin{array}{l}1251 . \\
1039 . \\
1311 . \\
1339 . \\
1041 . \\
1246 . \\
1542 . \\
1329 . \\
1238 . \\
1124 . \\
14430 \\
1440 . \\
1339 . \\
911 .\end{array}$ & $\begin{array}{l}.356 \\
.345 \\
.377 \\
.368 \\
.361 \\
.340 \\
.312 \\
.395 \\
.265 \\
.309 \\
.349 \\
.400 \\
.445 \\
.381\end{array}$ & $\begin{array}{r}349 . \\
536 . \\
2400 . \\
352 . \\
2330 . \\
412 . \\
2203 . \\
2100 . \\
249 . \\
142 . \\
415 . \\
3 . \\
708 . \\
2350 .\end{array}$ \\
\hline
\end{tabular}

\begin{tabular}{|c|c|c|c|c|c|c|c|c|}
\hline DATE & $\begin{array}{l}\text { SHORT } \\
\text { WAUE } \\
\text { TOTAL }\end{array}$ & $\begin{array}{l}\text { SHORT } \\
\text { UAVE } \\
\text { MAXIMUM }\end{array}$ & $\begin{array}{l}\text { TIME OF } \\
\text { SHORT } \\
\text { MAXIYUH }\end{array}$ & $\begin{array}{l}\text { LONG } \\
\text { WAUE } \\
\text { TOTAL }\end{array}$ & $\begin{array}{l}\text { LOHG } \\
\text { WAVE } \\
\text { MAXIMUH }\end{array}$ & $\begin{array}{l}\text { TIME OF } \\
\text { LDNG } \\
\text { MAXIMUH }\end{array}$ & $\begin{array}{l}\text { LONG } \\
\text { WAUE } \\
\text { MIHIMUM }\end{array}$ & $\begin{array}{l}\text { TIME OF } \\
\text { LOHG } \\
\text { MIMIMUH }\end{array}$ \\
\hline 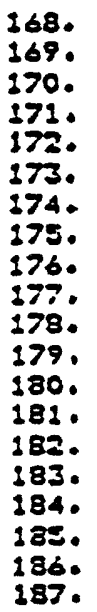 & $\begin{array}{l}722.5 \\
615.6 \\
581.8 \\
378.9 \\
331.9 \\
670.2 \\
710.5 \\
384.8 \\
629.0 \\
473.1 \\
689.4 \\
697.2 \\
84.6 \\
482.8 \\
640.2 \\
264.7 \\
219.6 \\
353.7 \\
721.0 \\
678.0\end{array}$ & $\begin{array}{l}1.578 \\
1.593 \\
1.421 \\
1.366 \\
1.653 \\
1.447 \\
1.352 \\
1.400 \\
1.435 \\
1.503 \\
1.474 \\
1.375 \\
1.396 \\
1.687 \\
1.549 \\
1.721 \\
1.270 \\
1.641 \\
1.557 \\
1.417\end{array}$ & $\begin{array}{l}1129 . \\
1230 . \\
11180 \\
1257 . \\
14220 \\
1249 . \\
1234 . \\
1246 . \\
1234 . \\
1350 . \\
1227 . \\
1159 . \\
1350 . \\
1032 . \\
1359 . \\
1107 . \\
1543 . \\
1336 . \\
1039 . \\
1245 .\end{array}$ & $\begin{array}{r}694.8 \\
743.9 \\
796.7 \\
315.4 \\
771.9 \\
740.2 \\
807.6 \\
806.7 \\
775.3 \\
642.6 \\
635.4 \\
782.0 \\
777.1 \\
733.9 \\
805.9 \\
796.6 \\
721.8 \\
632.3 \\
749.3\end{array}$ & $\begin{array}{l}.585 \\
.597 \\
.624 \\
.625 \\
.631 \\
.575 \\
.606 \\
.635 \\
.646 \\
.535 \\
.510 \\
.575 \\
.601 \\
.589 \\
.632 \\
.596 \\
.582 \\
.480 \\
.577\end{array}$ & $\begin{array}{r}1255 . \\
1656 . \\
1348 . \\
1629 . \\
237 . \\
2400 . \\
1311 . \\
1457 . \\
1009 . \\
5230 \\
1350 . \\
2306 . \\
1201 . \\
1105 . \\
1338 . \\
930 . \\
1336 . \\
631 . \\
1551 .\end{array}$ & $\begin{array}{l}.391 \\
.440 \\
.505 \\
.329 \\
.451 \\
.472 \\
.505 \\
.505 \\
.442 \\
.379 \\
.372 \\
.440 \\
.449 \\
.458 \\
.432 \\
.501 \\
.368 \\
.336 \\
.456\end{array}$ & $\begin{array}{r}3 . \\
300 . \\
2216 . \\
7 . \\
2223 . \\
709 . \\
137 . \\
157 . \\
2204 . \\
2239 . \\
159 . \\
2 . \\
2312 . \\
2141 . \\
17 . \\
2006 . \\
2346 . \\
2 . \\
3 .\end{array}$ \\
\hline
\end{tabular}

\begin{tabular}{|c|c|c|c|c|c|c|c|c|}
\hline DATE & $\begin{array}{l}\text { SHERT } \\
\text { HAVE } \\
\text { TOTAL }\end{array}$ & $\begin{array}{l}\text { SHORT } \\
\text { UAUE } \\
\text { MAXIYUM }\end{array}$ & $\begin{array}{l}\text { TIHE OF } \\
\text { SHORT } \\
\text { MAXINUM }\end{array}$ & $\begin{array}{l}\text { LONE } \\
\text { WAVE } \\
\text { TOTAL }\end{array}$ & $\begin{array}{l}\text { LOHO } \\
\text { WNVE } \\
\text { MAXIMUH }\end{array}$ & $\begin{array}{l}\text { TIME OF } \\
\text { LOHG } \\
\text { MAXIMUH }\end{array}$ & $\begin{array}{l}\text { LONE } \\
\text { WAVE } \\
\text { MINIMUM }\end{array}$ & $\begin{array}{l}\text { TIME OF } \\
\text { LONG } \\
\text { МIHIMUK }\end{array}$ \\
\hline $\begin{array}{l}188 . \\
299 . \\
190 . \\
19: . \\
192 . \\
193 . \\
194 . \\
195 . \\
196 . \\
197 . \\
198 . \\
199 . \\
200 .\end{array}$ & $\begin{array}{l}417.2 \\
660.3 \\
618.9 \\
605.1 \\
625.7 \\
695.3 \\
657.0 \\
623.1 \\
527.0 \\
399.4 \\
265.8 \\
463.7 \\
446.4\end{array}$ & $\begin{array}{l}1.705 \\
1.343 \\
1.977 \\
1.310 \\
1.637 \\
1.336 \\
1.278 \\
1.273 \\
1.7=3 \\
1.340 \\
.800 \\
1.829 \\
1.361\end{array}$ & $\begin{array}{l}1240 . \\
1222 . \\
1240 . \\
1203 . \\
1340 . \\
1248 . \\
1122 . \\
1237 . \\
1308 . \\
1+13 . \\
819 . \\
1320 . \\
1008 .\end{array}$ & $\begin{array}{l}905.5 \\
879.6 \\
758.1 \\
801.0 \\
724.5 \\
744.2 \\
797.4 \\
814.5 \\
804.8 \\
810.9 \\
774.3 \\
803.5 \\
791.8\end{array}$ & $\begin{array}{l}.638 \\
.052 \\
.653 \\
.608 \\
.585 \\
.563 \\
.634 \\
.026 \\
.039 \\
.653 \\
.600 \\
.629 \\
.613\end{array}$ & $\begin{array}{l}:=01 . \\
1=25 . \\
: 320 . \\
1307 . \\
1630 . \\
1401 . \\
1257 . \\
1347 . \\
1309 . \\
1424 . \\
1158 . \\
1=30 . \\
: 358 .\end{array}$ & $\begin{array}{l}.49: \\
.515 \\
.310 \\
.503 \\
.447 \\
.449 \\
.468 \\
.515 \\
.501 \\
.479 \\
.479 \\
.478 \\
.503\end{array}$ & $\begin{array}{r}209 . \\
455 . \\
2357 . \\
350 . \\
2356 . \\
15 . \\
312 . \\
456 . \\
413 . \\
2215 . \\
21 \pm 2 . \\
2235 . \\
2=25 .\end{array}$ \\
\hline
\end{tabular}


Table 6. Land station: (1) Daily total short- and long-wave radiation; (2) daily maximum short-wave radiation and the time it occurred; and (3) daily maximum and minimum long-wave radiation and the time they occurred. [continued]

\begin{tabular}{|c|c|c|c|c|c|c|c|c|}
\hline DATE & $\begin{array}{l}\text { SHORT } \\
\text { WAUE } \\
\text { TOTAL }\end{array}$ & $\begin{array}{l}\text { SHORT } \\
\text { UAvE } \\
\text { MnXIMUM }\end{array}$ & $\begin{array}{l}\text { TIME OF } \\
\text { SHORT } \\
\text { MnXIMUM }\end{array}$ & $\begin{array}{l}\text { LONG } \\
\text { WAVE } \\
\text { TOTAL }\end{array}$ & $\begin{array}{l}\text { Loug } \\
\text { WnYE } \\
\text { MXIMUM }\end{array}$ & $\begin{array}{l}\text { TIME OF } \\
\text { LONG } \\
\text { MAXIMUK }\end{array}$ & $\begin{array}{l}\text { LONG } \\
\text { WHUE } \\
\text { MINIMUM }\end{array}$ & $\begin{array}{l}\text { TIME OF } \\
\text { LONG } \\
\text { HIMIMUM }\end{array}$ \\
\hline $\begin{array}{l}201 . \\
202 . \\
203 . \\
204 . \\
205 . \\
206 . \\
207 . \\
208 . \\
209 . \\
210 . \\
218 . \\
212 . \\
213 . \\
214 . \\
215 . \\
215 .\end{array}$ & $\begin{array}{l}363.5 \\
314.2 \\
663.4 \\
598.5 \\
345.0 \\
649.5 \\
507.4 \\
301.2 \\
458.4 \\
589.8 \\
319.5 \\
551.9 \\
648.1 \\
180.4 \\
591.1 \\
812.1\end{array}$ & $\begin{array}{l}1.799 \\
1.433 \\
1.515 \\
1.748 \\
1.776 \\
1.366 \\
1.614 \\
1.039 \\
1.436 \\
1.497 \\
1.689 \\
1.717 \\
1.300 \\
1.287 \\
1.473 \\
1.395\end{array}$ & 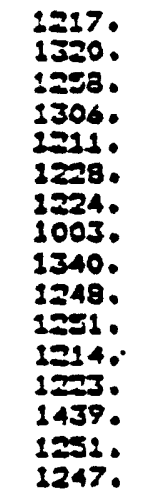 & $\begin{array}{l}828.4 \\
876.1 \\
768.9 \\
743.2 \\
747.1 \\
736.1 \\
775.5 \\
826.0 \\
827.2 \\
782.8 \\
808.0 \\
734.1 \\
739.1 \\
816.1 \\
826.3 \\
803.1\end{array}$ & $\begin{array}{l}.641 \\
.671 \\
.613 \\
.613 \\
.622 \\
.659 \\
.645 \\
.643 \\
.636 \\
.636 \\
.639 \\
.603 \\
.039 \\
.617 \\
.643 \\
.618\end{array}$ & $\begin{array}{l}1939 . \\
1417 . \\
1838 . \\
1306 . \\
1339 . \\
656 . \\
1348 . \\
1444 . \\
1340 . \\
1326 . \\
15380 \\
1214 . \\
1337 . \\
1439 . \\
1359 . \\
1303 .\end{array}$ & $\begin{array}{l}.535 \\
.545 \\
.468 \\
.466 \\
.454 \\
.461 \\
.461 \\
.515 \\
.521 \\
.486 \\
.472 \\
.452 \\
.472 \\
.519 \\
.524 \\
.507\end{array}$ & $\begin{array}{r}1 . \\
2358 . \\
2348 . \\
2211 . \\
2131 . \\
2231 . \\
338 . \\
323 . \\
2305 . \\
2004 . \\
2348 . \\
2153 . \\
2104 . \\
201 . \\
123 . \\
2139 .\end{array}$ \\
\hline
\end{tabular}

\begin{tabular}{|c|c|c|c|c|c|c|c|c|}
\hline DATE & $\begin{array}{l}\text { SHORT } \\
\text { WAUE } \\
\text { TOTAL }\end{array}$ & $\begin{array}{l}\text { SHORT } \\
\text { WAUE } \\
\text { MAXIMUM }\end{array}$ & $\begin{array}{l}\text { TIME OF } \\
\text { SHORT } \\
\text { MAXIMUM }\end{array}$ & $\begin{array}{l}\text { LONG } \\
\text { WAUE } \\
\text { TOTAL }\end{array}$ & $\begin{array}{l}\text { - ONG } \\
\text { WAUE } \\
\text { MAXIMUS }\end{array}$ & $\begin{array}{l}\text { TIME OF } \\
\text { LONE } \\
\text { MAXIMUM }\end{array}$ & $\begin{array}{l}\text { LONG } \\
\text { WAVE } \\
\text { KINIMUH }\end{array}$ & $\begin{array}{l}\text { TIME OF } \\
\text { LONG } \\
\text { MINIMUM }\end{array}$ \\
\hline $\begin{array}{l}217 . \\
218 . \\
219 . \\
220 . \\
221 . \\
222 . \\
223 . \\
224 . \\
220 . \\
226 . \\
227 . \\
229 . \\
229 . \\
230 .\end{array}$ & $\begin{array}{l}579.2 \\
623.0 \\
617.0 \\
621.9 \\
131.9 \\
372.5 \\
623.2 \\
607.2 \\
518.2 \\
596.4 \\
196.7 \\
201.2 \\
530.5 \\
370.9\end{array}$ & $\begin{array}{l}1.418 \\
1.268 \\
1.262 \\
1.294 \\
1.041 \\
1.713 \\
1.324 \\
1.303 \\
1.438 \\
1.247 \\
1.398 \\
1.099 \\
1.526 \\
1.606\end{array}$ & $\begin{array}{l}1: 55 . \\
1214 . \\
1216 . \\
1214 . \\
12040 \\
1230 . \\
1217 . \\
12040 \\
1527 . \\
12120 \\
1154 . \\
1413 . \\
1129 . \\
1129 .\end{array}$ & $\begin{array}{l}853.7 \\
753.4 \\
800.0 \\
712.8 \\
796.3 \\
777.5 \\
699.9 \\
743.6 \\
778.0 \\
746.4 \\
799.0 \\
790.2 \\
809.6 \\
824.5\end{array}$ & $\begin{array}{l}.638 \\
.575 \\
.611 \\
.584 \\
.592 \\
.627 \\
.529 \\
.556 \\
.624 \\
.570 \\
.003 \\
.596 \\
.622 \\
.631\end{array}$ & $\begin{array}{l}1518 . \\
1240 . \\
2208 . \\
132 . \\
1205 . \\
1325 . \\
1272 . \\
1517 . \\
1430 . \\
1616 . \\
1601 . \\
1856 . \\
91.0 \\
1534 .\end{array}$ & $\begin{array}{l}.524 \\
.466 \\
.300 \\
.445 \\
.300 \\
.445 \\
.435 \\
.470 \\
.484 \\
.473 \\
.494 \\
.010 \\
.494 \\
.489\end{array}$ & $\begin{array}{r}240 . \\
541 . \\
2357 . \\
2125 . \\
31 . \\
2400 . \\
2052 . \\
811 . \\
2338 . \\
2204 . \\
538 . \\
2022 . \\
2317 . \\
111 .\end{array}$ \\
\hline
\end{tabular}

\begin{tabular}{|c|c|c|c|c|c|c|c|c|}
\hline MATE & $\begin{array}{l}\text { SHOKT } \\
\text { WAVE } \\
\text { TOTAL }\end{array}$ & $\begin{array}{c}\text { SHORT } \\
\text { WAUE } \\
\text { MAXTMUn }\end{array}$ & $\begin{array}{l}\text { TIME OF } \\
\text { SHORT } \\
\text { MiXIMUH }\end{array}$ & $\begin{array}{l}\text { LOLE } \\
\text { WA'E } \\
\text { TOTAL }\end{array}$ & $\begin{array}{l}\text { LonG } \\
\text { WnuE } \\
\text { mXIMUM }\end{array}$ & $\begin{array}{c}\text { TIME OF } \\
\text { LDHB } \\
\text { MnXIMISH }\end{array}$ & $\begin{array}{l}\text { LONG } \\
\text { UNUE } \\
\text { MINIMUM }\end{array}$ & $\begin{array}{l}\text { TIME OF } \\
\text { LONG } \\
\text { MINIMUM }\end{array}$ \\
\hline $\begin{array}{l}231 . \\
2320 \\
2330 \\
2340 \\
2350 \\
236 . \\
237 . \\
238 . \\
239 . \\
240 . \\
241 . \\
242 . \\
=43 . \\
244 .\end{array}$ & $\begin{array}{l}435.7 \\
322.0 \\
246.8 \\
461.6 \\
492.9 \\
264.0 \\
448.9 \\
479.5 \\
437.7 \\
477.3 \\
299.9 \\
523.3 \\
316.0 \\
494.3\end{array}$ & $\begin{array}{l}1.494 \\
1.733 \\
1.467 \\
1.466 \\
1.421 \\
1.263 \\
1.274 \\
1.431 \\
1.573 \\
1.193 \\
.784 \\
1.134 \\
1.051 \\
1.214\end{array}$ & $\begin{array}{l}1420 . \\
1153 . \\
1138 . \\
1231 . \\
1234 . \\
1337 . \\
1217 . \\
1=40 . \\
1206 . \\
1139 . \\
12030 \\
1=27 . \\
1=20 . \\
1300 .\end{array}$ & $\begin{array}{l}729.4 \\
754.9 \\
806.6 \\
741.6 \\
772.6 \\
835.4 \\
050.5 \\
780.8 \\
803.6 \\
303.1 \\
748.1 \\
756.0 \\
795.1 \\
776.0\end{array}$ & $\begin{array}{l}.618 \\
.591 \\
.655 \\
.564 \\
.608 \\
.655 \\
.678 \\
.629 \\
.015 \\
.634 \\
.084 \\
.001 \\
.639 \\
.608\end{array}$ & $\begin{array}{r}29, \\
2351 . \\
1157 . \\
1231 . \\
2630 . \\
1427 . \\
1505 . \\
9 . \\
1556 . \\
1547 . \\
1724 . \\
300 . \\
1401 . \\
1430 .\end{array}$ & $\begin{array}{l}.409 \\
.473 \\
.440 \\
.456 \\
.477 \\
.510 \\
.473 \\
.482 \\
.508 \\
.489 \\
.505 \\
.482 \\
.484 \\
.479\end{array}$ & $\begin{array}{r}2008 . \\
152 . \\
2339 . \\
17 . \\
727 . \\
258 . \\
4330 \\
450 . \\
2=24 . \\
501 . \\
2359 . \\
2156 . \\
=400 . \\
333 .\end{array}$ \\
\hline
\end{tabular}


Table 6. Land station: (1) Daily total short- and long-wave radiation; (2) daily maximum short-wave radiation and the time it occurred; and (3) daily maximum and minimum long-wave radiation and the time they occurred. [continued]

\begin{tabular}{|c|c|c|c|c|c|c|c|c|}
\hline DATE & $\begin{array}{l}\text { SHORT } \\
\text { WAVE } \\
\text { TOTAL }\end{array}$ & $\begin{array}{c}\text { SHORT } \\
\text { UAUE } \\
\text { MAXIMUM }\end{array}$ & $\begin{array}{l}\text { TTME OF } \\
\text { SHORT } \\
\text { MAXIMUH }\end{array}$ & $\begin{array}{l}\text { LOHE } \\
\text { WAUE } \\
\text { TOTAL }\end{array}$ & $\begin{array}{l}\text { LONG } \\
\text { WAIJE } \\
\text { HAXIMUM }\end{array}$ & $\begin{array}{l}\text { TIHE OF } \\
\text { LDNE } \\
\text { MAXIMUM }\end{array}$ & $\begin{array}{l}\text { LONE } \\
\text { UAUE } \\
\text { MIMIMUM }\end{array}$ & $\begin{array}{l}\text { TIME OF } \\
\text { LONE } \\
\text { AINEMU }\end{array}$ \\
\hline $\begin{array}{l}245 . \\
246 . \\
247 . \\
248 . \\
249 . \\
250 . \\
251 . \\
252 . \\
253 . \\
254 . \\
253 . \\
256 . \\
257 . \\
253 . \\
259 . \\
250 . \\
251 . \\
262 .\end{array}$ & $\begin{array}{r}476.1 \\
134.0 \\
129.3 \\
378.7 \\
266.7 \\
467.7 \\
415.2 \\
373.3 \\
446.8 \\
83.8 \\
368.3 \\
344.6 \\
275.3 \\
38.7 \\
96.3 \\
186.6 \\
460.8 \\
128.9\end{array}$ & $\begin{array}{r}1.104 \\
1.201 \\
1.045 \\
1.303 \\
1.224 \\
1.129 \\
1.232 \\
1.414 \\
1.187 \\
.1633 \\
1.361 \\
1.376 \\
1.048 \\
.147 \\
.678 \\
1.096 \\
1.071 \\
.463\end{array}$ & $\begin{array}{r}1239 . \\
10180 \\
9160 \\
1310 . \\
1133 . \\
12310 \\
1117 . \\
12310 \\
1224 . \\
1109 . \\
1327 . \\
1329 . \\
1112 . \\
1447 . \\
1239 . \\
1251 . \\
1141 . \\
1308 .\end{array}$ & $\begin{array}{l}824.0 \\
780.3 \\
789.4 \\
747.8 \\
698.3 \\
710.6 \\
796.6 \\
781.7 \\
676.1 \\
671.4 \\
657.8 \\
629.3 \\
690.3 \\
740.8 \\
714.8 \\
747.3 \\
579.6 \\
670.6\end{array}$ & $\begin{array}{l}.529 \\
.010 \\
.603 \\
.578 \\
.540 \\
.540 \\
.634 \\
.596 \\
.592 \\
.505 \\
.538 \\
.515 \\
.521 \\
.522 \\
.522 \\
.556 \\
.493 \\
.491\end{array}$ & $\begin{array}{r}1320 . \\
11 . \\
1521 . \\
234 . \\
1133 . \\
817 . \\
2000 . \\
1046 . \\
331 . \\
1209 . \\
1332 . \\
1335 . \\
1448 . \\
947 . \\
1259 . \\
1324 . \\
1 . \\
1308 .\end{array}$ & $\begin{array}{l}.314 \\
.472 \\
.303 \\
.437 \\
.400 \\
.426 \\
.459 \\
.489 \\
.375 \\
.365 \\
.407 \\
.372 \\
.428 \\
.301 \\
.405 \\
.449 \\
.353 \\
.388\end{array}$ & $\begin{array}{r}242 . \\
2210 . \\
11 . \\
2301 . \\
812 . \\
423 . \\
20 . \\
2026 . \\
2400 . \\
229 . \\
1045 . \\
2040 . \\
110 . \\
37 . \\
2316 . \\
8 . \\
2102 . \\
128 .\end{array}$ \\
\hline
\end{tabular}

\begin{tabular}{|c|c|c|c|c|c|c|c|c|}
\hline DATE & $\begin{array}{l}\text { SHORT } \\
\text { WAVE } \\
\text { TOTAL }\end{array}$ & $\begin{array}{l}\text { SHDRT } \\
\text { WAUE } \\
\text { MAXIMUM }\end{array}$ & $\begin{array}{l}\text { TIME OF } \\
\text { SHIORT } \\
\text { MAXIMUM }\end{array}$ & $\begin{array}{l}\text { LONG } \\
\text { WAVE } \\
\text { TOTAL }\end{array}$ & $\begin{array}{l}\text { LOME } \\
\text { HAVE } \\
\text { MAXIMUM }\end{array}$ & $\begin{array}{l}\text { TIME OF } \\
\text { LONG } \\
\text { MXXIMUM }\end{array}$ & $\begin{array}{l}\text { LOHS } \\
\text { WAUE } \\
\text { MINIMUM }\end{array}$ & $\begin{array}{l}\text { TIME OF } \\
\text { LOHAO } \\
\text { MINSMUH }\end{array}$ \\
\hline $\begin{array}{l}263 . \\
264 . \\
265 . \\
266 . \\
267 . \\
203 . \\
269 . \\
270 . \\
270^{\circ} \\
272 . \\
273 . \\
274 . \\
275 . \\
276 . \\
277 . \\
278 . \\
279 . \\
280 . \\
281 . \\
282 .\end{array}$ & $\begin{array}{r}196.0 \\
249.4 \\
190.1 \\
445.3 \\
373.0 \\
368.8 \\
103.4 \\
361.6 \\
206.3 \\
80.1 \\
74.7 \\
135.2 \\
79.1 \\
89.0 \\
122.5 \\
186.6 \\
365.0 \\
44.6 \\
362.3 \\
236.4\end{array}$ & $\begin{array}{r}1.264 \\
1.426 \\
.999 \\
1.035 \\
1.288 \\
.373 \\
.977 \\
.978 \\
1.084 \\
.269 \\
.652 \\
1.083 \\
.768 \\
.427 \\
1.301 \\
1.213 \\
.957 \\
.326 \\
.924 \\
1.191\end{array}$ & $\begin{array}{l}1231 . \\
1152 . \\
1017 . \\
1225 . \\
1251 . \\
1257 . \\
1138 . \\
1153 . \\
1210 . \\
1026 . \\
1119 . \\
1301 . \\
1125 . \\
1330 . \\
1246 . \\
1123 . \\
1258 . \\
1258 . \\
1218 . \\
1216 .\end{array}$ & $\begin{array}{l}633.2 \\
631.4 \\
608.7 \\
558.1 \\
601.3 \\
665.0 \\
645.7 \\
705.4 \\
778.2 \\
732.5 \\
778.8 \\
777.2 \\
737.6 \\
712.3 \\
706.6 \\
636.9 \\
588.3 \\
660.5 \\
549.9 \\
619.2\end{array}$ & $\begin{array}{r}.475 \\
.487 \\
.465 \\
.438 \\
.533 \\
.524 \\
.489 \\
.575 \\
.613 \\
.522 \\
.584 \\
.573 \\
.538 \\
.510 \\
.510 \\
.505 \\
.477 \\
.498 \\
.449 \\
.519\end{array}$ & $\begin{array}{r}1339 . \\
1321 . \\
1021 . \\
730 . \\
1244 . \\
1346 . \\
1316 . \\
1451 . \\
1317 . \\
2400 . \\
1341 . \\
22 . \\
1127 . \\
3 . \\
1250 . \\
1125 . \\
2229 . \\
1257 . \\
733 . \\
1145 .\end{array}$ & $\begin{array}{l}.349 \\
.354 \\
.314 \\
.346 \\
.372 \\
.421 \\
.414 \\
.432 \\
.486 \\
.432 \\
.301 \\
.468 \\
.300 \\
.375 \\
.424 \\
.328 \\
.321 \\
.363 \\
.353 \\
.365\end{array}$ & 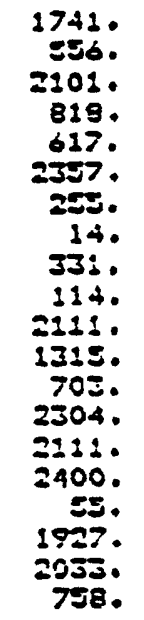 \\
\hline
\end{tabular}

\begin{tabular}{|c|c|c|c|c|c|c|c|c|}
\hline DATE & $\begin{array}{l}\text { SHORT } \\
\text { WAVE } \\
\text { TOTAL }\end{array}$ & $\begin{array}{l}\text { SiroRT } \\
\text { WAUE } \\
\text { MAXIMUM }\end{array}$ & $\begin{array}{l}\text { TIME OF } \\
\text { SHORT } \\
\text { MAXIMUH }\end{array}$ & $\begin{array}{l}\text { LONG } \\
\text { UAVE } \\
\text { TOTAL }\end{array}$ & 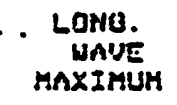 & $\begin{array}{l}\text { TIME OF } \\
\text { LONG } \\
\text { MnXIMUY }\end{array}$ & $\begin{array}{l}\text { LONG } \\
\text { WA'EE } \\
\text { MIHIMUM }\end{array}$ & $\begin{array}{l}\text { TIME OF } \\
\text { LOHG } \\
\text { MINIMUM }\end{array}$ \\
\hline $\begin{array}{l}293 . \\
294 . \\
299 . \\
284 . \\
207 . \\
288 . \\
289 . \\
290 . \\
291 . \\
292 . \\
293 . \\
294 . \\
295 . \\
296 . \\
29 \% . \\
298 . \\
299 .\end{array}$ & $\begin{array}{r}22.2 \\
340.9 \\
244.1 \\
104.0 \\
337.5 \\
37.6 \\
: 34.8 \\
275.8 \\
45.1 \\
41.4 \\
179.7 \\
93.0 \\
: 37.9 \\
105.6 \\
51.4 \\
220.5 \\
235.3\end{array}$ & $\begin{array}{l}.104 \\
.893 \\
.901 \\
.391 \\
.368 \\
.225 \\
1.074 \\
1.082 \\
.336 \\
.277 \\
1.036 \\
.414 \\
.995 \\
.416 \\
.412 \\
.953 \\
.794\end{array}$ & $\begin{array}{l}1=33 . \\
1234 . \\
1210 . \\
1244 . \\
1202 . \\
1252 . \\
1134 . \\
12=2 . \\
1051 . \\
12=2 . \\
11470 \\
1226 . \\
1129 . \\
1=310 \\
1416 . \\
1=00 . \\
1: 53 .\end{array}$ & $\begin{array}{l}720.3 \\
556.0 \\
533.7 \\
625.0 \\
535.0 \\
664.8 \\
621.4 \\
610.0 \\
659.9 \\
676.4 \\
624.8 \\
679.9 \\
630.0 \\
630.8 \\
616.6 \\
434.0 \\
5=3.4\end{array}$ & $\begin{array}{l}.529 \\
.029 \\
.45: \\
.443 \\
.454 \\
.482 \\
.485 \\
.495 \\
.475 \\
.486 \\
.491 \\
.001 \\
.482 \\
.481 \\
.000 \\
.460 \\
.435\end{array}$ & $\begin{array}{r}2358 . \\
134 . \\
2147 . \\
1209 . \\
36 . \\
2100 . \\
357 . \\
1421 . \\
1205 . \\
1241 . \\
1144 . \\
1320 . \\
620 . \\
1334 . \\
1412 . \\
1251 . \\
145.0\end{array}$ & $\begin{array}{l}.475 \\
.302 \\
.312 \\
.433 \\
.305 \\
.253 \\
.310 \\
.347 \\
.437 \\
.407 \\
.351 \\
.393 \\
.367 \\
.316 \\
.309 \\
.304 \\
.335\end{array}$ & $\begin{array}{r}57 . \\
2 \pm 57 . \\
1114 . \\
609 . \\
644 . \\
4 . \\
2935 . \\
829 . \\
1=6 . \\
250 . \\
2053 . \\
1741 . \\
1956 . \\
1528 . \\
2546 . \\
133 . \\
1=9 .\end{array}$ \\
\hline
\end{tabular}

